\title{
Peering into the Dark Side: Magnesium Lines Establish a Massive Neutron Star in PSR J2215+5135
}

\author{
M. Linares ${ }^{1,2}$ (i), T. Shahbaz ${ }^{2,3}$, and J. Casares ${ }^{2,3}$ (1) \\ ${ }^{1}$ Departament de Física, EEBE, Universitat Politècnica de Catalunya, c/ Eduard Maristany 10, E-08019 Barcelona, Spain \\ ${ }^{2}$ Instituto de Astrofísica de Canarias, c/ Vía Láctea s/n, E-38205 La Laguna, Tenerife, Spain \\ ${ }^{3}$ Universidad de La Laguna, Departamento de Astrofísica, E-38206 La Laguna, Tenerife, Spain \\ Received 2018 January 9; revised 2018 April 6; accepted 2018 April 10; published 2018 May 23
}

\begin{abstract}
New millisecond pulsars (MSPs) in compact binaries provide a good opportunity to search for the most massive neutron stars. Their main-sequence companion stars are often strongly irradiated by the pulsar, displacing the effective center of light from their barycenter and making mass measurements uncertain. We present a series of optical spectroscopic and photometric observations of PSR J2215+5135, a "redback" binary MSP in a 4.14 hr orbit, and measure a drastic temperature contrast between the dark/cold $\left(T_{\mathrm{N}}=5660_{-380}^{+260} \mathrm{~K}\right)$ and bright/hot $\left(T_{\mathrm{D}}=8080_{-280}^{+470} \mathrm{~K}\right)$ sides of the companion star. We find that the radial velocities depend systematically on the atmospheric absorption lines used to measure them. Namely, the semi-amplitude of the radial velocity curve (RVC) of J2215 measured with magnesium triplet lines is systematically higher than that measured with hydrogen Balmer lines, by $10 \%$. We interpret this as a consequence of strong irradiation, whereby metallic lines dominate the dark side of the companion (which moves faster) and Balmer lines trace its bright (slower) side. Further, using a physical model of an irradiated star to fit simultaneously the two-species RVCs and the three-band light curves, we find a center-of-mass velocity of $K_{2}=412.3 \pm 5.0 \mathrm{~km} \mathrm{~s}^{-1}$ and an orbital inclination $i=63^{\circ} .9_{-2.7}^{+2.4}$. Our model is able to reproduce the observed fluxes and velocities without invoking irradiation by an extended source. We measure masses of $M_{1}=2.27_{-0.15}^{+0.17} M_{\odot}$ and $M_{2}=0.33_{-0.02}^{+0.03} M_{\odot}$ for the neutron star and the companion star, respectively. If confirmed, such a massive pulsar would rule out some of the proposed equations of state for the neutron star interior.
\end{abstract}

Key words: binaries: general - pulsars: general - pulsars: individual (PSR J2215+5135) - stars: neutron - stars: variables: general - X-rays: binaries

\section{Introduction}

New millisecond pulsars (MSPs) in compact binaries (orbital period $P_{\text {orb }} \lesssim 1$ day) are being discovered with the advent of the Fermi Large Area Telescope (LAT; Atwood et al. 2009). Their companion or secondary stars are light $\left(\sim 0.1 M_{\odot}\right.$ in the so-called redbacks) or ultralight $\left(\sim 0.01 M_{\odot}\right.$ in the black widows), and in some cases they are strongly irradiated by the pulsar wind and high-energy radiation powered by the neutron star's rotational energy loss $(\dot{E})$. Furthermore, 3 of the nearly 20 known redback MSPs have shown transitions between the radio-pulsar and accretion-disk states, which has provided a long-sought link between MSPs and low-mass X-ray binaries (LMXBs; Archibald et al. 2009; de Martino et al. 2013; Papitto et al. 2013; see Linares 2014 for a review of redback states).

Most of these new compact binary MSPs are relatively nearby $(\lesssim 4 \mathrm{kpc})$ and far from the Galactic plane $\left(\gtrsim 5^{\circ}\right)$, where interstellar extinction is low. This allows for sensitive optical spectroscopic observations and dynamical studies of the companion star in its orbit around the pulsar, and offers a new opportunity to measure the mass of spun-up "recycled" neutron stars (e.g., Romani \& Shaw 2011; Crawford et al. 2013; Kaplan et al. 2013). However, as we discuss in the present work, the effects of irradiation on the measured radial velocities must be carefully taken into account in order to avoid systematic uncertainties.

PSR J2215+5135 (J2215 hereafter) was discovered as a $2.61 \mathrm{~ms}$ MSP in radio searches of the LAT source 1FGL J2216.1+5139 (i.e., 2 FGL J2215.7+5135 or 3FGL J2215.6+5134), and to-date has the shortest $P_{\text {orb }}$ among Galactic field redbacks $\left(P_{\text {orb }} \simeq 4.14 \mathrm{hr}\right.$; Hessels et al. 2011). Even though this system has been observed so far only in the (disk-free, rotation powered) pulsar state, Linares (2014) found a relatively high X-ray luminosity $L_{\mathrm{X}} \sim 10^{32} \mathrm{erg} \mathrm{s}^{-1}$, suggesting $\mathrm{J} 2215$ as a candidate for future transitions to an accreting state. Optical photometry revealed a $V=20.2-18.7$ mag counterpart with orbital variability typical of strongly irradiated systems (Breton et al. 2013; Schroeder \& Halpern 2014). Modeling the optical light curves (LCs) can determine the inclination of the orbit that, together with the precise ephemerides obtained from pulsar timing, may allow a full orbital solution and a neutron star mass measurement (e.g., van Kerkwijk et al. 2011; see also Shahbaz et al. 1998; Casares et al. 2006). Nevertheless, the orbital parameters for J2215 presented by Schroeder \& Halpern 2014 (based on LC model fits) and by Romani et al. (2015 including also radial velocities) differ by a large amount, yielding inconsistent neutron star masses $M_{\mathrm{NS}}$ in the range 1.6-2.5 $M_{\odot}$.

We present here the results of a new set of observations of J2215 taken in 2014 with three different telescopes (Section 2), including the $10.4 \mathrm{~m}$ Gran Telescopio Canarias (GTC). These reveal an extreme temperature contrast between the cold/dark ("night") and hot/bright ("day") faces of the secondary star (Section 3.1). In order to place tighter independent constraints on $M_{\mathrm{NS}}$ and to investigate systematic effects on dynamical studies of this new class of pulsars, we carefully measure the spectral type and radial velocity of the companion along the orbit (Section 2.3).

We find that the apparent radial velocities of J2215 depend on both the spectral range and the reference/template spectrum 
Table 1

Summary of Optical Observations of PSR J2215+5135

\begin{tabular}{|c|c|c|c|c|c|c|c|c|}
\hline $\begin{array}{l}\text { Telescope } \\
\text { (diameter) }\end{array}$ & $\begin{array}{l}\text { Instrument } \\
\text { (configuration) }\end{array}$ & $\begin{array}{l}\text { Band }^{\mathrm{a}} \\
(\lambda, \AA)\end{array}$ & $\begin{array}{c}\text { Date } \\
\text { (evening) }\end{array}$ & $\begin{array}{l}\text { Time } \\
\text { (UT) }\end{array}$ & $\begin{array}{c}\text { Exposures } \\
(\mathrm{nr} . \times \text { duration })\end{array}$ & $\begin{array}{l}\text { Orbital } \\
\text { Phase }\end{array}$ & Airmass & $\begin{array}{c}\text { Seeing } \\
\left({ }^{\prime \prime}\right)\end{array}$ \\
\hline \multicolumn{9}{|c|}{ Photometry } \\
\hline $\begin{array}{l}\text { IAC- } 80 \\
(80 \mathrm{~cm})\end{array}$ & $\begin{array}{l}\text { CAMELOT } \\
(\text { bin } 2 \times 2)\end{array}$ & $\begin{array}{c}g^{\prime}, r^{\prime} \\
(4639,6122)\end{array}$ & 2014 Aug 02 & 22:53-04:01 & $\begin{array}{c}4 \times 420 \mathrm{~s} \\
+26 \times 600 \mathrm{~s}\end{array}$ & $0.4-1.6$ & $1.47-1.09$ & $1.1-2.5$ \\
\hline $\begin{array}{l}\text { IAC- } 80 \\
(80 \mathrm{~cm})\end{array}$ & $\begin{array}{l}\text { CAMELOT } \\
(\text { bin } 2 \times 2)\end{array}$ & $\begin{array}{c}g^{\prime}, r^{\prime} \\
(4639,6122)\end{array}$ & 2014 Aug 03 & $23: 32-03: 52$ & $30 \times 420 s$ & $0.3-1.3$ & $1.32-1.09$ & $0.4-0.7$ \\
\hline $\begin{array}{l}\text { WHT } \\
(4.2 \mathrm{~m})\end{array}$ & $\begin{array}{c}\text { ACAM } \\
\text { (win. } 501 \times 501)\end{array}$ & $\begin{array}{c}g^{\prime}, r^{\prime}, i^{\prime} \\
(4639,6122,7439)\end{array}$ & 2014 Aug 11 & $22: 15-00: 03$ & $95 \times 60 \mathrm{~s}$ & $0.4-0.8$ & $1.48-1.18$ & $0.7-1.6$ \\
\hline $\begin{array}{l}\text { WHT } \\
(4.2 \mathrm{~m})\end{array}$ & $\begin{array}{c}\text { ACAM } \\
(\text { win. } 501 \times 501)\end{array}$ & $\begin{array}{c}g^{\prime}, r^{\prime}, i^{\prime} \\
(4639,6122,7439)\end{array}$ & 2014 Sep 01 & 23:17-02:06 & $149 \times 60 \mathrm{~s}$ & $0.4-1.0$ & $1.13-1.09$ & $0.8-1.4$ \\
\hline \multicolumn{9}{|c|}{ Spectroscopy } \\
\hline $\begin{array}{l}\text { WHT } \\
(4.2 \mathrm{~m})\end{array}$ & $\begin{array}{c}\text { ISIS } \\
(\mathrm{R} 600 \mathrm{~B}+\mathrm{R})\end{array}$ & $\begin{array}{l}\text { B:3700-5300 } \\
\text { R:5500-7200 }\end{array}$ & 2014 Aug 11 & 00:23-05:19 & $17 \times 900 \mathrm{~s}$ & $0.0-1.1$ & $1.17-1.33$ & $0.7-1.6$ \\
\hline $\begin{array}{l}\text { GTC } \\
(10.4 \mathrm{~m})\end{array}$ & $\begin{array}{l}\text { OSIRIS } \\
\text { (R2000B) }\end{array}$ & B:4000-5700 & 2014 Nov 14 & $19: 47-21: 06$ & $5 \times 935 \mathrm{~s}$ & $0.6-0.8$ & $1.09-1.11$ & $0.6-1.1$ \\
\hline $\begin{array}{l}\text { GTC } \\
(10.4 \mathrm{~m})\end{array}$ & $\begin{array}{l}\text { OSIRIS } \\
\text { (R2000B) }\end{array}$ & B:4000-5700 & 2014 Nov 15 & $19: 43-23: 58$ & $16 \times 935 \mathrm{~s}$ & $0.3-1.3$ & $1.09-1.55$ & $0.7-1.3$ \\
\hline
\end{tabular}

Note.

${ }^{\text {a }}$ Effective wavelength of the photometric filters or approximate wavelength range covered by the spectra, in Angstroms.

used to measure them (Section 3.2). In Section 4, we model jointly the observed LCs and radial velocities, including for the first time dynamical information of the cold/dark side of the companion. We find a new orbital solution (Section 4.2) with an extremely massive neutron star (Section 5.3). We discuss these results in Section 5, as well as the implications for dynamical studies in compact binaries with strong irradiation. Section 6 contains a summary of our main results and conclusions.

\section{Observations, Reduction, and Analysis}

\subsection{Photometry}

We obtained phase-resolved photometric observations of PSR J2215+5135 with the IAC-80 and William Herschel (WHT) telescopes at the Teide and Roque de los Muchachos observatories, respectively, on four different nights (see Table 1). The IAC-80 images were taken on 2014 August 2-3 with the Teide observatory light improved camera (CAMELOT; 0.30 arcsec pixel $^{-1}$ ) using the SDSS filters $g^{\prime}$ and $r^{\prime}$, an exposure time of either $420 \mathrm{~s}$ or $600 \mathrm{~s}$ and a binning of $2 \times 2$ pixels in order to optimize the signal-to-noise ratio $(\mathrm{S} / \mathrm{N})$. The WHT images were taken on 2014 August 11 (contemporaneous with our WHT-ISIS spectra) and September 1, with the auxiliary-port camera (ACAM; 0.25 arcsec pixel $^{-1}$ in imaging mode) using the SDSS filters $g^{\prime}, r^{\prime}$ and $i^{\prime}$, an exposure time of $60 \mathrm{~s}$ and a window of $501 \times 501$ pixels around the source in order to reduce readout time (readout and filter change resulted in deadtime of 6-9 s per exposure).

All images were debiased and flat-fielded using standard IRAF routines. We then performed aperture photometry using the ULTRACAM pipeline, a variable source extraction radius (set to 1.5-1.7 times the seeing) and a nearby stable nonsaturated reference star. The resulting differential magnitudes are relative to a nearby star in the field and thus insensitive to thin clouds or moderate atmospheric variability (yet the observing conditions were generally good). The absolute flux (apparent magnitude) calibration was done using a nearby AAVSO-APASS star, with uncertainties of $0.05 \mathrm{mag}\left(r^{\prime}\right)$ and $0.1 \mathrm{mag}\left(g^{\prime}, i^{\prime}\right)$, and checked against other nearby stars from the USNO-B1 catalog. We also compared these reference star magnitudes with those given by the PANSTARRS catalog and found only a significant difference in the $r^{\prime}$ band, with a shift of +0.16 mag with respect to the APASS values that we use. We verified that this has no impact on any of the results reported in this work; in particular, the parameters reported in Section 4 are all consistent within the errors when using the PANSTARRS instead of the APASS calibration. Figure 1 (top) shows the J2215 LCs folded at the orbital period.

\subsection{Spectroscopy}

We observed J2215 with the WHT and GTC telescopes on 2014 August 11 and November 14-15, respectively, in order to obtain medium resolution spectra of the companion star and measure its spectral type and velocity along the orbit. For the WHT-ISIS spectra, we chose the R600B (blue arm) and R600R (red arm) gratings centered at $4500 \AA$ and $6400 \AA$, respectively. The slit width was set to $1^{\prime \prime}$, resulting into a resolution of $105-130 \mathrm{~km} \mathrm{~s}^{-1}(R \sim 2600)$ and $65-80 \mathrm{~km} \mathrm{~s}^{-1}(R \sim 4000)$ for the blue and red arms, respectively. At the GTC, we used OSIRIS in its long-slit spectroscopy mode, with the R2000B VPH gratings and a slit width of $1^{\prime \prime}$, resulting in a resolution of $145-160 \mathrm{~km} \mathrm{~s}^{-1}(R \sim 2000)$. With this campaign we obtained 17 WHT-ISIS and 21 GTC-OSIRIS spectra covering the full $4.14 \mathrm{hr}$ orbit with some redundancy and with exposure times of $900 \mathrm{~s}$ and $935 \mathrm{~s}$, respectively (i.e., exposing each spectrum for about $6 \%$ of the $\mathrm{J} 2215$ orbit; see Table 1). 


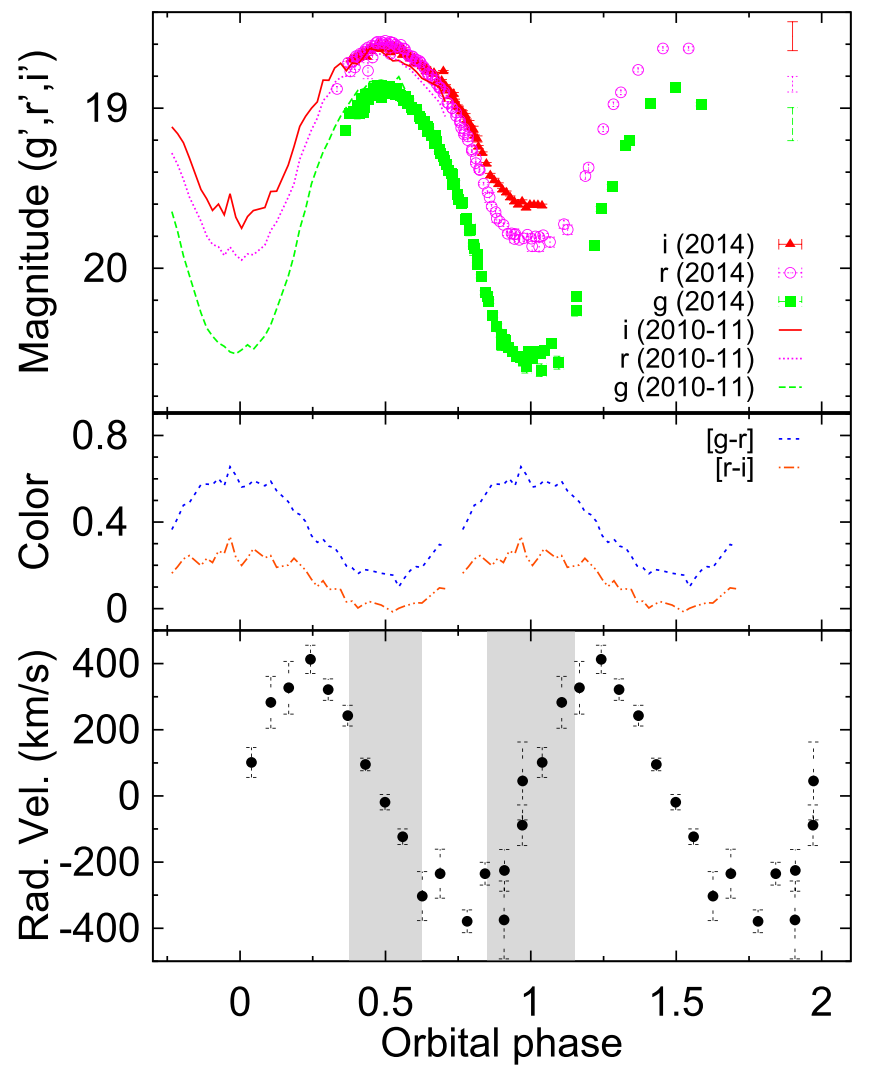

Figure 1. Top: optical light curves of J2215 in three bands, as indicated. Data points show our 2014 IAC-80 and WHT observing campaign and lines (solid, dotted, and dashed) show the 2010-2011 results from Schroeder \& Halpern (2014). Error bars in the top right corner show the uncertainty on the magnitude calibration (Section 2.1; errors on differential magnitude are plotted but smaller than the symbols). Middle: color variation along the orbit showing redder/ colder emission around light minimum (orbital phase 0). Bottom: radial velocity curve from our WHT-ISIS spectra, calculated by cross-correlating the full spectra (red and blue arms) with an F5 template. The averages used for optimal subtraction are shown with gray shaded rectangles (Section 2.3).

After applying bias and flat corrections to the trimmed images within IRAF, we extracted the spectra and subtracted sky background using the optimal extraction method within STARLINK/PAMELA to account for the significant tilt (Marsh 1989). The WHT-ISIS spectra were calibrated in wavelength using interspersed arc spectra ( $\mathrm{CuNe}, \mathrm{CuAr}$ ) extracted from the same source extraction regions, taken once every two source spectra. A set of well identified arc lines were satisfactorily fitted with a fourth-order polynomial to produce the wavelength scale (47 and 31 lines in the blue and red arms, resulting in an rms amplitude of residuals of $0.05 \AA$ and $0.02 \AA$, respectively). We adjusted the same polynomial function to all arc spectra and interpolated in time between adjacent arcs to calibrate the science spectra, thereby accounting for the significant $(\sim 1 \AA)$ drift due to instrument flexure. The GTC-OSIRIS wavelength calibration was done using one set of arcs taken on the second night, fitting the pixel-wavelength relation with a fourth-order polynomial (19 lines giving residuals with an rms amplitude of $0.06 \AA$ ). The resulting wavelength calibration was checked and refined using the O I sky line at $5577 \AA$, which allowed us to correct for residual $\left(\lesssim 10 \mathrm{~km} \mathrm{~s}^{-1}\right)$ shifts in the wavelength solution.

\subsection{Spectral Analysis}

In order to measure the radial velocity and temperature of the companion star in J2215 throughout the orbit, we applied within MOLLY the cross-correlation and optimal subtraction techniques, respectively. As both techniques require comparison spectra (or templates hereafter), we built a library of mainsequence stellar templates with spectral types between $\mathrm{O} 4$ and M0 (see the Appendix). The continuum level of all 38 source and 33 template spectra was normalized with a spline fit, and subtracted.

After binning to a same heliocentric velocity scale, excluding telluric lines and broadening the template spectra to the source spectral resolution (Section 2.2), each source and template spectra were cross-correlated to find their relative velocity, allowing shifts between -700 and $+700 \mathrm{~km} \mathrm{~s}^{-1}$. The resulting radial velocity curves (RVCs) from the GTC and WHT spectra were fitted with a sine function $V=G+$ $K \sin \left[2 \pi\left(t-T_{0}\right) / P_{\text {orb }}\right]$, where $V$ and $G$ are the radial and systemic velocities, $K$ is the semi-amplitude of the RVC, $t$ is the middle time of each spectrum, $T_{0}$ is the time of inferior conjunction of the secondary that defines ${ }^{4}$ orbital phase $\Phi_{\text {orb }}=0$ and $P_{\text {orb }}$ is the orbital period. Having checked that the best-fit period is consistent with (but less precise than) the orbital period from pulsar timing (Abdo et al. 2013), this parameter was subsequently fixed at the pulsar timing value $P_{\text {orb }}=0.1725021049[8]$ days.

Using the orbital parameters above, we corrected for the systemic velocity and orbital motion and shifted all source spectra to the reference frame of each template. In order to increase the $\mathrm{S} / \mathrm{N}$, we averaged four to six source spectra around $\Phi_{\text {orb }}=0 \pm 0.15$ and $\Phi_{\text {orb }}=0.5 \pm 0.125$. We then performed an optimal subtraction using the full GTC spectral range, i.e., subtracted the templates scaled by a factor $f_{\text {veil }}$ from the source averaged spectra, adjusting $f_{\text {veil }}$ to minimize the residual scatter. This is a quantitative way of matching the observed absorption lines from J2215 to a set of templates with known spectral types and temperatures (Marsh et al. 1994).

Because J2215 becomes very faint around $\Phi_{\text {orb }}=0$ ( $r \simeq 20$ mag; Figure 1, where the cold face of the companion star dominates), the corresponding ISIS spectra have low S/Ns. The tightest constraints on the spectral type and radial velocities come from the higher S/N GTC spectra, so we focus the rest of our analysis on those. Only 1 out of the 21 GTC spectra could not be included in the analysis due to the very low ( $<100$ at peak) number of counts collected.

Thanks to the large GTC collecting area, we were able to measure radial velocities using (i) the full spectral range (4000-5300 $\AA$ ), (ii) the hydrogen Balmer lines (three $50 \AA$ wide windows centered on $\mathrm{H} \beta, \mathrm{H} \gamma$ and $\mathrm{H} \delta$ ), and (iii) the MgI triplet lines present in the J2215 spectra (5152-5199 ^). The corresponding spectral ranges and two representative GTC-OSIRIS spectra are shown in Figure 3. As the absorption spectra of early/hot and late/cold stars are dominated by different sets of lines (see, e.g., the Appendix), the Balmer and $\mathrm{Mg}$ I radial velocities allow us to track different parts of the irradiated companion throughout its orbit around the pulsar.

The cross-correlation of two broad lines may yield ambiguous results if the profiles are not exactly the same.

\footnotetext{
4 Note different definition than usual pulsar timing phase zero, which marks the time of the ascending node of the pulsar.
} 


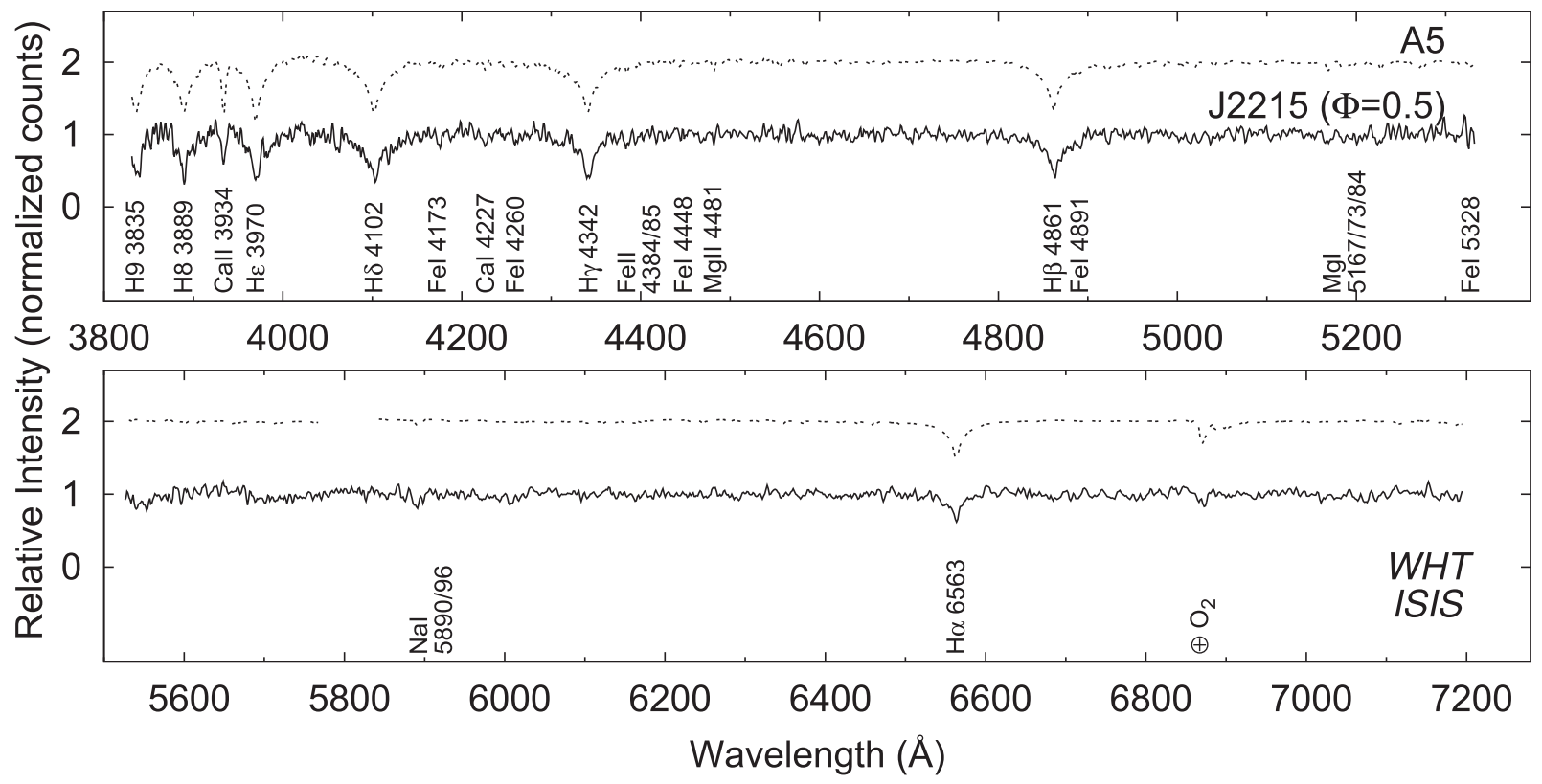

Figure 2. Normalized WHT-ISIS spectra of (from bottom up, arbitrary shifts for display purpose) J2215 around orbital phase 0.5 (solid line) and an A5 spectral type standard star (dotted line). The main absorption lines are identified along the bottom axes.

The matching wings can give two maxima in the crosscorrelation function, at velocities that differ from that measured using the line core ("double peaked" cross-correlation functions). On close inspection of J2215's RVCs, we found that this introduces strong artificial deviations from a sinusoidal function around phase 0.5 , only when using Balmer lines and templates of spectral type earlier than F (i.e., when both source and template spectra are dominated by broad lines). For this reason, we include only spectral types later than F0 in our results for the Balmer-line RVCs (Section 3.2).

\section{Results}

\subsection{Temperatures of the Hot and Cold Sides}

The optical flux from J2215 varies smoothly along the orbit (no flares are detected in the $60 \mathrm{~s}$ cadence data), with one clear maximum and minimum per orbital cycle and a peak-to-peak amplitude of almost two magnitudes $\left(g^{\prime}, B\right.$ bands). We find that the orbital LCs (Figure 1) are stable over timescales of years, comparing our 2014 observations with the 2010-2011 data presented by Schroeder \& Halpern (2014; converting their $B V R$ magnitudes into the SDSS $g^{\prime} r^{\prime} i^{\prime}$ system following Jordi et al. 2006). The $\sim 0.4 \mathrm{mag}$ change in the $\left[g^{\prime}-r^{\prime}\right]$ color reveals hotter emission at maximum light $\left(\Phi_{\text {orb }}=0.5\right)$. The RVC of the companion shows a large amplitude $\left(K \sim 400 \mathrm{~km} \mathrm{~s}^{-1}\right)$ and changes sign near the maximum and minimum light ( $\Phi_{\mathrm{orb}}=0.5$ and 0 , respectively), as can be seen already from the WHT spectra (Figure 1) and as first reported by Romani et al. (2015). These properties are indicative of a companion star that is strongly irradiated by the pulsar throughout the compact $4.14 \mathrm{hr}$ orbit.

The J2215 spectra around $\Phi_{\text {orb }}=0.5$ (Figures 2 and 3) show strong Balmer lines (H1 through H9) consistent with an A5 star, as well as numerous yet weaker metallic $(\mathrm{Ca} / \mathrm{Fe} / \mathrm{Mg})$ lines. Around $\Phi_{\text {orb }}=0$, when the companion star presents its cold face to the observer, Balmer lines are much weaker and narrower, while $\mathrm{MgI}$ triplet lines are stronger. Hence the equivalent widths (EW) of Balmer and $\mathrm{MgI}$ lines are anticorrelated along the orbit, as shown in Figure 4. The optimal subtraction analysis (Section 2.3) gives a clean measurement of the temperature and spectral type of the companion star, independent of the measured colors (which may be contaminated by nonstellar light). We find drastic changes between the irradiated and cold sides of the companion star. This is shown qualitatively in Figures 3 and 2, where the J2215 GTC and WHT spectra are compared to A5 and G5 templates degraded to the same resolution.

Figure 5 shows our quantitative results: the reduced chisquared resulting from the optimal subtraction method (Section 2.3) for templates with a broad range of spectral types $(\mathrm{O}-\mathrm{M})$. We thereby measure a spectral type $\mathrm{A} 5 \pm 2$ for the brightest spectra (i.e., A3-A7 at $\Phi_{\text {orb }}=0.5$ ) and G5 \pm 5 at the faintest end $\left(\mathrm{G} 0-\mathrm{K} 0\right.$ at $\Phi_{\mathrm{orb}}=0$; Romani et al. 2015 report similar yet slightly earlier spectral types of A2 and G0 around phases 0.5 and 0 , respectively, but no errors are quoted). These correspond to effective temperatures for the cold ("night") and hot ("day") sides of $T_{\mathrm{N}}=5660_{-380}^{+260} \mathrm{~K}$ and $T_{\mathrm{D}}=8080_{-280}^{+470} \mathrm{~K}$, respectively (Pecaut \& Mamajek 2013). We thus find a drastic temperature contrast between opposite sides of the companion star, where the hot/day side is about $2400 \mathrm{~K}$ or $40 \%$ hotter than it would be without irradiation. The best matching scale factors are $f_{\text {veil }} \simeq 0.8$ at both superior and inferior conjunction, suggesting a contribution from nonstellar light (veiling) of about $20 \%$ in this GTC-OSIRIS $4000-5300 \AA$ band (which corresponds approximately to filter $g^{\prime}$ ).

\subsection{Radial Velocities: Magnesium versus Balmer Lines}

We find that the radial velocities and $K$ values depend systematically on the set of lines or spectral range used to measure them. Namely, as we show in Figure 6, the semiamplitude of the $\mathrm{Mg}$ I line $\mathrm{RVC}$ ( $K_{\mathrm{Mg}}$, red circles) is always $\sim 10 \%$ higher than the semi-amplitude of the Balmer-line RVC ( $K_{\mathrm{Balmer}}$, blue squares). Using the same G5 template yields $K_{\text {Balmer }}=382.8 \pm 4.7 \mathrm{~km} \mathrm{~s}^{-1}$ and $K_{\mathrm{Mg}}=420.2 \pm 6.2 \mathrm{~km} \mathrm{~s}^{-1}$. 


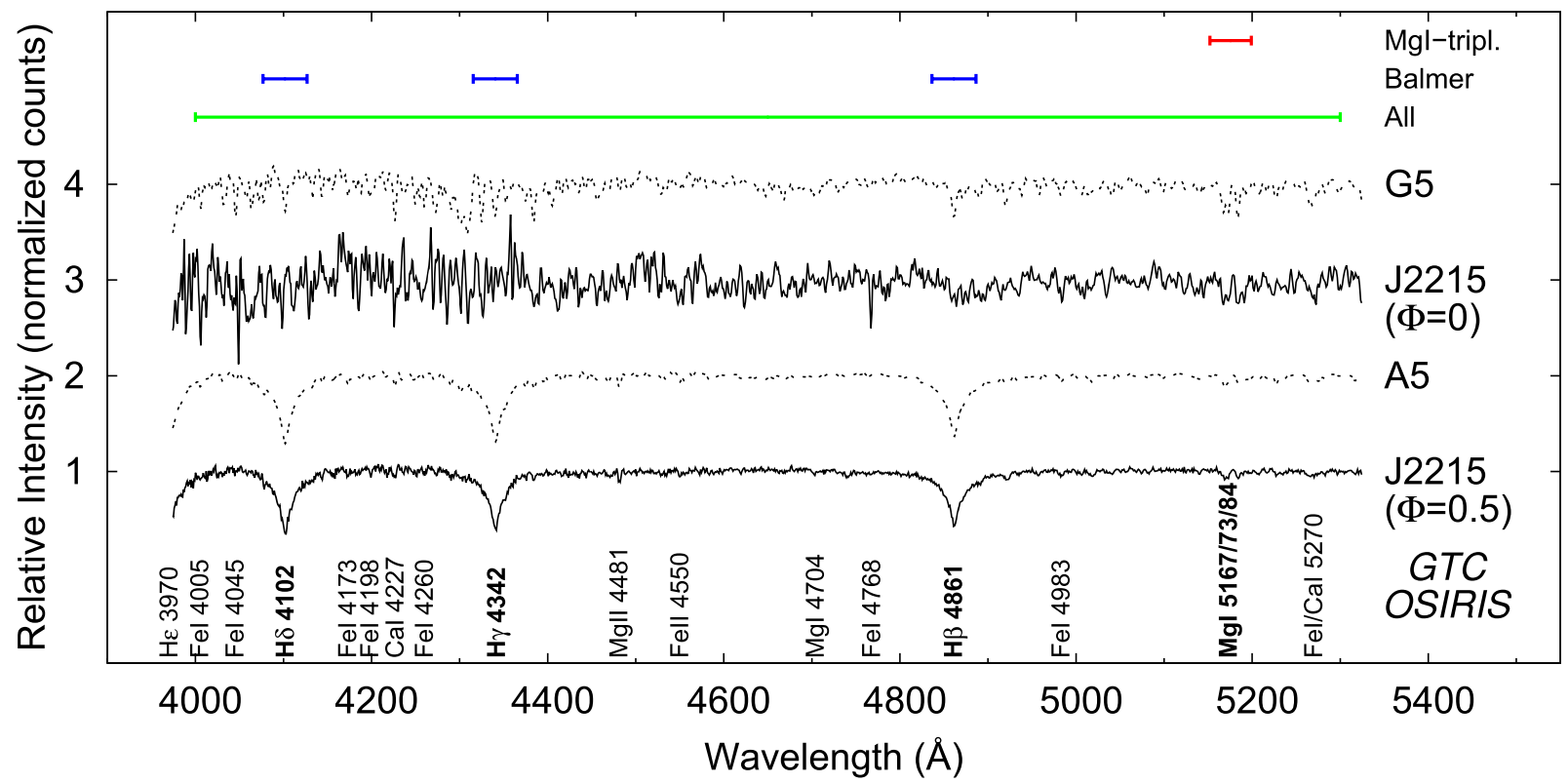

Figure 3. Normalized GTC-OSIRIS spectra of (from bottom up, arbitrary shifts for display purpose): J2215 around orbital phase 0.5 (i.e., companion at superior conjunction; solid line); an A5 spectral type standard star (dotted line); J2215 around orbital phase 0 (companion at inferior conjunction; solid line); and a G5 spectral type standard star (dotted line). The main absorption lines are identified along the bottom axis. Green/blue/red lines along the top axis show the ranges used for radial velocity measurements (Section 2.3).

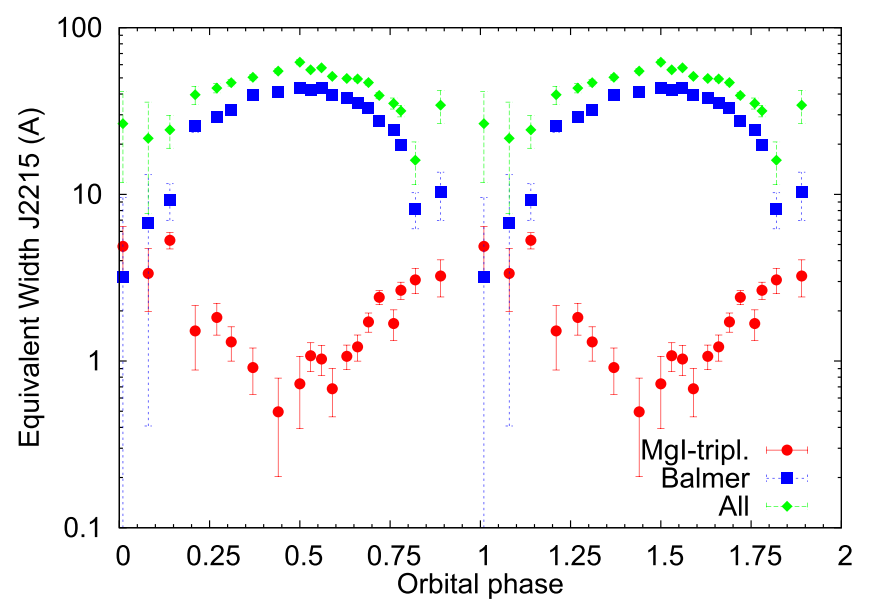

Figure 4. Equivalent width (EW) of all (green diamonds), Balmer (blue squares), and Mg I (red circles) absorption lines in the GTC spectra of J2215, as a function of orbital phase.

In Figure 7, we present two extreme cases: RVCs of J2215 calculated using Balmer lines (blue symbols) and Mg I-triplet lines (red symbols; see Section 2.3 for the exact wavelength ranges). In both cases the radial velocities were measured by cross-correlating the J2215 spectra with the same G5 template. The Mg I triplet lines yield higher radial velocities than the Balmer lines (in absolute value) around orbital phases 0.25 and 0.75 , when the companion star is viewed sideways (in "quadrature"). Because the velocities at quadrature are extreme, this has an important impact on the measured $K$ values and the corresponding neutron star mass (Section 5.1).

Most RVCs are reasonably well fitted with a pure sinusoidal function (reduced chi-squared $\lesssim 2$ ). There are, however, deviations noticeable in the sine fit residuals (Figure 7), especially in the Balmer-line RVCs (spectral types earlier than F1 were not used in the Balmer-line cross-correlation analysis, see Section 2.3). The fitted $K$ values were verified in a model

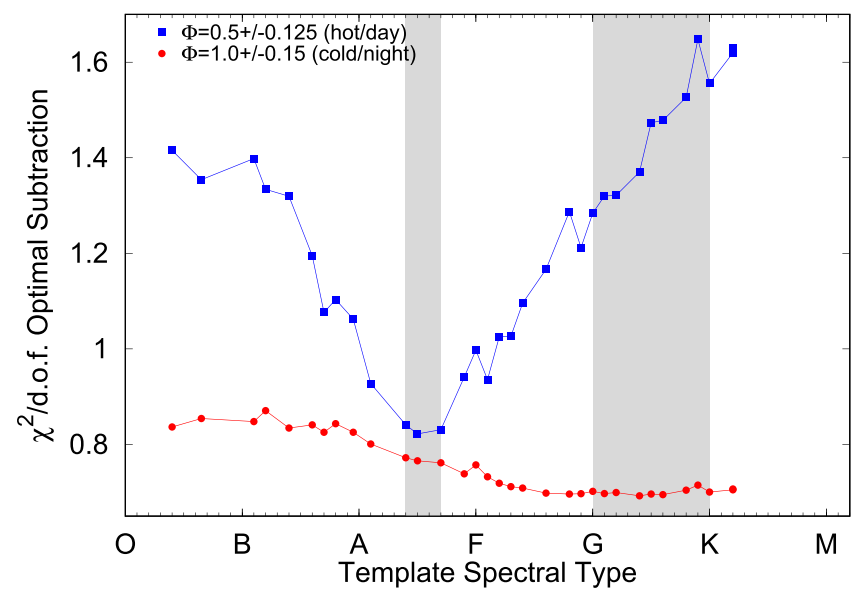

Figure 5. Reduced chi-squared of the optimal subtraction plotted vs. template spectral type. Minima show the spectral type of the optical companion to J2215 around orbital phase 0.5 (blue squares, left shaded region) and phase 1 (red circles, right shaded region; see Section 2.3).

independent way by measuring the peak to peak semiamplitude of the RVC: the two maximum and minimum radial velocities were averaged, subtracted and divided by two. The results were always consistent with the $K$ values presented in Figure 6.

Using the full ensemble of lines, on the other hand, yields intermediate values of the RVC semi-amplitude (green triangles in Figure 6; $K_{\mathrm{All}}=398.8 \pm 2.3 \mathrm{~km} \mathrm{~s}^{-1}$ for a G5 template). We also find a clear systematic dependence with the template's spectral type when using the full spectral range: a monotonic increase of $K_{\mathrm{All}}$ toward later (cooler) spectral types. We conclude that, when measuring radial velocities in strongly irradiated systems such as J2215, the spectral range and reference spectra must be chosen carefully in order to measure the pulsar mass accurately (see Section 5 for a further discussion). 


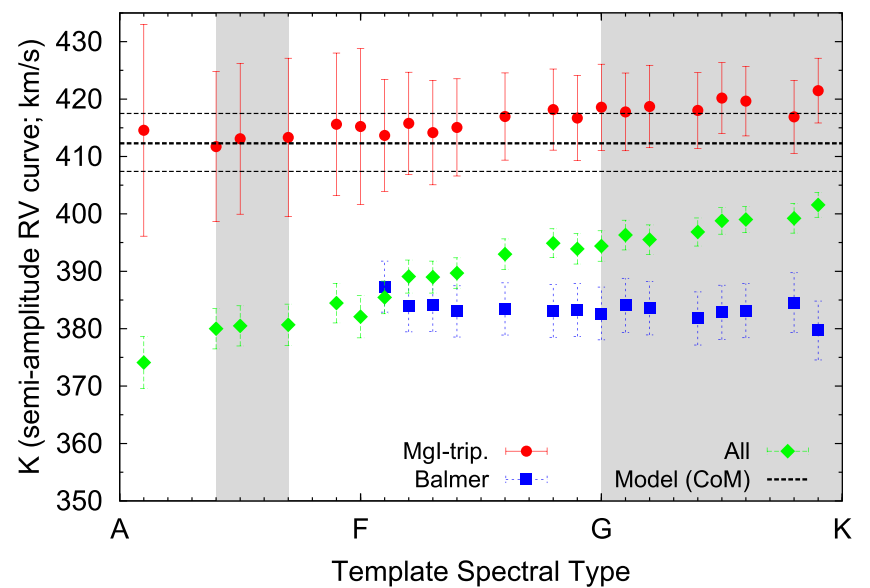

Figure 6. Semi-amplitude of the RVC of J2215 as a function of template spectral type, calculated using all absorption lines (green diamonds), Mg I lines (red circles), and Balmer lines (blue squares). Shaded regions show the spectral type of the hot/cold sides, and horizontal lines show the value of the center of mass semi-amplitude from our model (Section 4).

The systemic velocities that we find are all in the $40-70 \mathrm{~km} \mathrm{~s}^{-1}$ range, showing only small changes with spectral range or template spectral type. Averaging the results for spectral types G0-K0, we find $G=49.0 \pm 2.5$ (stat) \pm 8.0 (syst) $\mathrm{km} \mathrm{s}^{-1}$ (where the statistical and systematic errors correspond to the standard deviation of the full range and of all three spectral ranges, respectively). From the same sine fits, we find an epoch of zero phase (companion at inferior conjunction) of $\mathrm{T} 0=$ $56976.9501 \pm 0.0003$ (stat) \pm 0.0008 (syst) MJD (TDB), which we use together with the radio-pulsar timing $P_{\text {orb }}$ (Section 2.3) in order to compute orbital phases.

\section{Modeling}

In order to obtain the most reliable masses and orbital parameters, we modeled simultaneously the photometric threeband LCs and the Mg-triplet and Balmer-line RVCs, using the XRBCURVE model. The model, described in the following, has been successfully applied to LCs and RVCs of neutron star and black hole X-ray binaries (see Shahbaz et al. 2000, 2003, 2017 for more details).

\subsection{Binary Parameters and Irradiation}

XRBCURVE includes a nearly Roche-lobe-filling secondary star heated by high-energy photons from the compact object and an accretion disk (not included in this case since no disk emission lines are detected). The binary system's geometry is determined by the orbital inclination $i$, the mass ratio $q=M_{2} / M_{1}$ (where $M_{1}$ and $M_{2}$ are the masses of the neutron star and secondary star, respectively), and the Roche-lobe filling factor of the secondary star, $f$. The orbital period $P_{\text {orb }}$, the radial velocity semi-amplitude of the secondary star's barycenter $K_{2}$ and the distance to the source in kpc $(D)$ set the scale of the system. The light arising from the secondary star depends on its mean, unperturbed effective temperature $\left(T_{2}\right)$, and the gravity darkening exponent $\beta$.

The additional light due to irradiation is given by the irradiation efficiency $\eta \equiv L_{\text {irr }} / \dot{E}$, which we define in accordance with previous work as the ratio of the heating luminosity $\left(L_{\mathrm{irr}}\right.$, assuming isotropic emission by an irradiating point source at the compact object) to the spin-down luminosity of the pulsar $\left(\dot{E}=5.29 \times 10^{34} \mathrm{erg} \mathrm{s}^{-1}\right.$, Breton et al. 2013). We calculate the resulting increase in the local effective temperature due to the irradiating external source assuming that all irradiating flux is thermalized (Shahbaz et al. 2003). We use NEXTGEN modelatmosphere fluxes (Hauschildt et al. 1999) to determine the intensity distribution on the secondary star and a quadratic limb-darkening law with coefficients taken from Claret (2000), to correct the intensity. Based on the observed mid-G spectral type for the secondary star (Section 3), we fix $\beta$ at 0.10 (Lucy 1967).

To model the RVCs, we assume that the whole secondary star contributes to both Balmer and $\mathrm{Mg}$ I-triplet lines, including the inner/irradiated face. This is based on the corresponding EWs, which follow those expected from an A5-G5 star (Figure 4). We set the Balmer and Mg I-triplet absorption line strengths according to the effective temperature of each surface element of the star. Using our template spectra, we determine the EW versus temperature relationship for the same exact wavelength ranges used in measuring the RVCs (the Appendix; see Figure 13). The line strengths for each surface element on the star are then calculated using its temperature and the EWtemperature relation. Finally, radial velocities are calculated from the model line profile, averaged among all surface elements visible at each orbital phase.

In determining the binary parameters, we use a Markov chain Monte Carlo (MCMC) method convolved with a differential evolution fitting algorithm (see Vrugt 2016; Shahbaz et al. 2017). We use a Bayesian framework to determine our binary model parameters (Gregory 2005). We include the projected semimajor axis of the pulsar orbit measured from radio timing observations $\left(x_{1}=a_{1} \sin i=0.46814\right.$ [1] lt-s; Abdo et al. 2013) as an independent constraint on $q$ and $K_{2}\left(q=K_{2} P_{\text {orb }} / 2 \pi x_{1} c\right.$; where $c$ is the speed of light) using a Gaussian priori. Our MCMC fitting makes use of flat prior probability distributions for the rest of the model parameters. We use 20 individual chains to explore the parameter space and 40,000 iterations per chain. We reject the first 500 iterations and only include every tenth point.

We use a reddening of $E(B-V)=0.38$ mag, which we calculate from the X-ray absorbing column density toward J2215, $N_{\mathrm{H}}=2.1 \times 10^{21} \mathrm{~cm}^{-2}$ (Gentile et al. 2014) using the conversion from Predehl \& Schmitt (1995). Because $N_{\mathrm{H}}$ is in turn estimated from the measured pulsar dispersion measure (He et al. 2013), we verified the accuracy of the reddening toward J2215 in different ways. The total Galactic $N_{\mathrm{H}}$ in the direction of $\mathrm{J} 2215$ is $40 \%$ higher (Kalberla et al. 2005), although this may include additional absorbing material in the line of sight. Our value of $E(B-V)=0.38 \mathrm{mag}$ is consistent with that measured from IR dust maps in the same direction $(0.35 \pm 0.02 \mathrm{mag}$, Schlegel et al. 1998). In order to quantify the possible impact on the measured pulsar mass, we repeated the LC and RV fits with $E(B-V)$ left as a free parameter, with a flat priori. Reassuringly, this yields the same value $M_{1} \simeq 2.3 M_{\odot}$, and a value of $E(B-V)$ fully consistent with (less than $1 \sigma$ from) the one we use.

We also compute the line-of-sight temperature at orbital phases 0.0 and 0.5 , which represent the cold/night $\left(T_{\mathrm{N}}\right)$ and hot/day $\left(T_{\mathrm{D}}\right)$ temperatures of the secondary star. Using the spectral type measurements explained above, we impose temperature constraints on $T_{\mathrm{N}}(5280-5920 \mathrm{~K}$, corresponding to a spectral type $\mathrm{G} 5 \pm 5)$ and $T_{\mathrm{D}}(7800-8550 \mathrm{~K}$, corresponding 

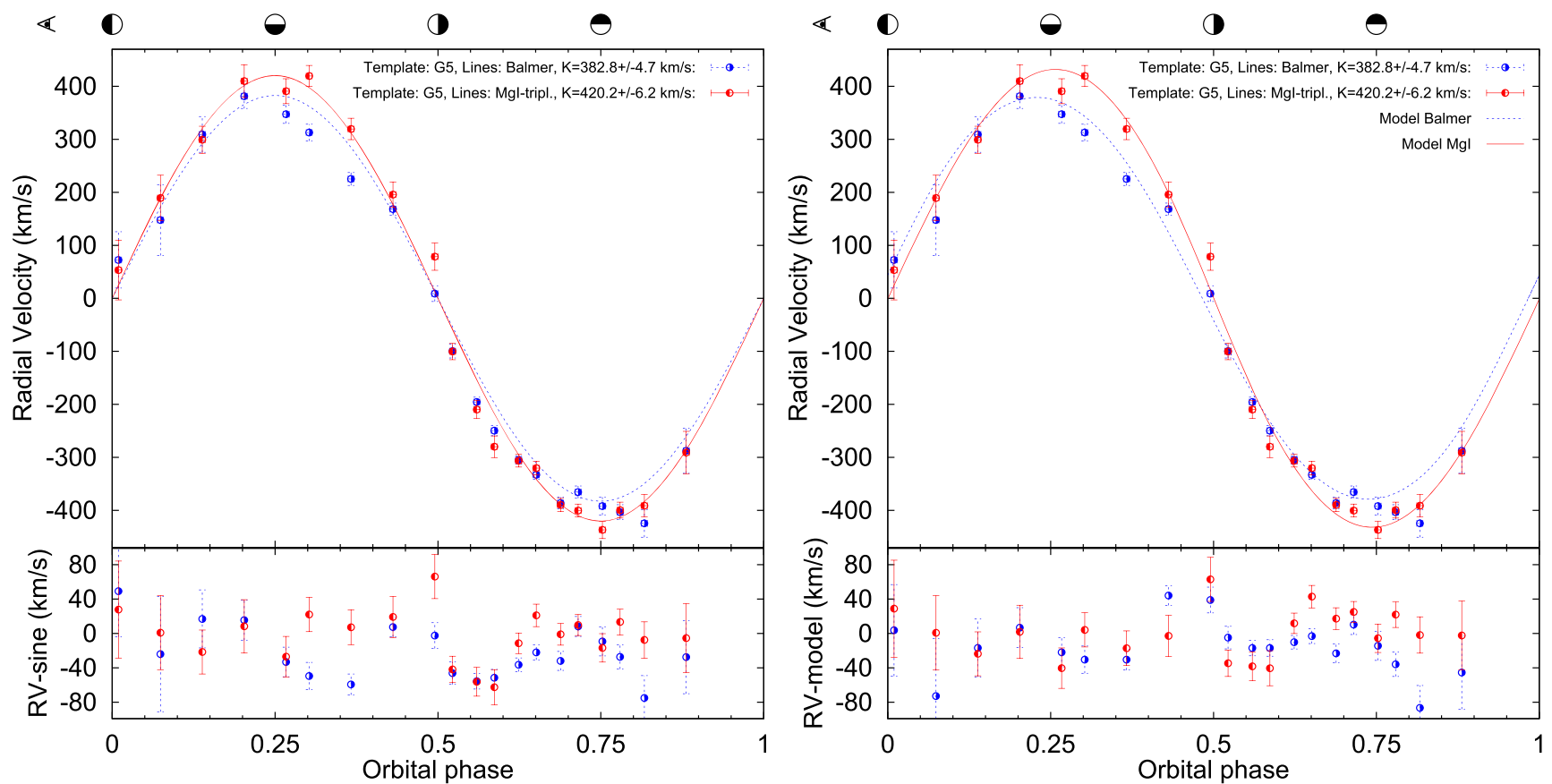

Figure 7. Radial velocity of the companion star in J2215 in its orbit around the pulsar, as measured by cross-correlation with a G5 template using (i) Balmer lines (blue points) and (ii) Mg I triplet lines (red points; Section 3.2). Left: blue dashed and red solid lines show the sinusoidal fits to the Balmer and Mg I RVCs, respectively (the best-fit systemic velocity was subtracted in both cases). Sine fit residuals are shown in the bottom panel. Right: best fit and residuals from our XRBCURVE model of a nearly Roche-lobe-filling irradiated star (Section 4).

Table 2

Comparison of Irradiated Binary Models for J2215

\begin{tabular}{|c|c|c|c|c|}
\hline Param. & This Work $^{\mathrm{a}}$ & $\mathrm{SH} 14^{\mathrm{b}}$ & $\mathrm{R} 15^{\mathrm{c}}$ & R16 ${ }^{\mathrm{d}}$ \\
\hline$f$ & $0.95_{-0.01}^{+0.01}$ & $1.00 \pm 0.01$ & $\simeq 1$ & $0.905 \pm 0.004$ \\
\hline$q$ & $0.144 \pm 0.002$ & $0.155-0.180$ & 0.145 & $?$ \\
\hline$i\left(^{\circ}\right)$ & $63.9_{-2.7}^{+2.4}$ & $49.5-54.3$ & 88.8 & $83 \pm 6$ \\
\hline$D(\mathrm{kpc})$ & $2.9 \pm 0.1$ & n.a. & $3.9 ?$ & $3.0 ?$ \\
\hline$K_{2}\left(\mathrm{~km} \mathrm{~s}^{-1}\right)$ & $412.3 \pm 5.0$ & $329-382^{\mathrm{e}}$ & $407.8^{\mathrm{f}}$ & $?$ \\
\hline$\eta$ & $2.9_{-0.2}^{+0.3}$ & $0.083 \pm 0.001$ & 0.97 & $2.16 \pm 0.15$ \\
\hline$M_{1}\left(M_{\odot}\right)$ & $2.27_{-0.15}^{+0.17}$ & $1.9-2.7$ & 1.59 & $?$ \\
\hline$M_{2}\left(M_{\odot}\right)$ & $0.33_{-0.02}^{+0.02}$ & $0.34-0.44$ & 0.23 & $?$ \\
\hline$T_{2}(\mathrm{~K})$ & $5630_{-71}^{+52}$ & $3765-3945$ & 6220 & $?$ \\
\hline$T_{\mathrm{N}}(\mathrm{K})$ & $5280-5920$ & n.a. & $>6000$ & $6416 \pm 58$ \\
\hline$T_{\mathrm{D}}(\mathrm{K})$ & $7800-8550$ & $4876-5090$ & $9264^{\mathrm{g}}$ & 9000 \\
\hline$\overline{\chi^{2} / \text { dof }}$ & $164 / 126=1.30$ & 1.5 & 4.4 & $1018 / 263=3.9$ \\
\hline
\end{tabular}

Notes. Ad-hoc constraints on the models are marked in italics.

${ }^{a}$ Our XRBCURVE fit to three-band photometric light curves $(g, r$ and $i$ ) and two-band spectroscopic radial velocity curves (Mg I and Balmer). See the text for details.

${ }^{\mathrm{b}}$ ELC fit to $B, V$, and $R$ light curves (Schroeder \& Halpern 2014, and references therein). Ranges from both their NextGen and PHOENIX models.

c ELC fit to the same three-band BVR light curves and one radial velocity curve (Romani et al. 2015). Their preferred "HiT" model (no errors reported).

d ICARUS fit to the same BVR LCs and RVC, with irradiation from an extended intrabinary shock and temperature constraint, from Romani \& Sanchez (2016). Their preferred "IBS-Td" model.

e Predicted from the LC model fit.

${ }^{\mathrm{f}}$ Derived from their best-fit $q, i$, and $M_{1}$.

${ }^{\mathrm{g}}$ Derived from their best-fit $M_{1}, M_{2}$, and $L_{\text {irr. }}$.

to a spectral type $\mathrm{A} 5 \pm 2$ ). Comparing with previous models of J2215 (Table 2), we conclude that our quantitative independent constraints on $T_{\mathrm{N}}$ and $T_{\mathrm{D}}$ are critical in order to find a good solution. From our measured $f_{\text {veil }} \simeq 0.8$ (Section 3.1), it is clear that there is an extra light component that veils the observed light from the secondary star. To allow for this wavelengthdependent veiling, we include an extra flux component in the model LCs, $f_{g}, f_{r}$, and $f_{i}$ in the $g, r$, and $i$ band, respectively. We also allow for possible uncertainties in the absolute flux calibration of the light curves, by including a wavelengthdependant magnitude offset in the same bands.

The model parameters that determine the shape and amplitude of the optical LCs and RVCs are $i, T_{2}, \eta, D$, and $K_{2}$ and the extra flux components $f_{g}, f_{r}$, and $f_{i}$. There are also a number of extra parameters: the phase shift for the LCs and RVCs (discussed below) as well as the systemic velocities for the Mg I-triplet and the Balmer-line RVCs (which we set to be the same). The $g^{\prime}, r^{\prime}, i^{\prime}$-band LCs were phase folded and 


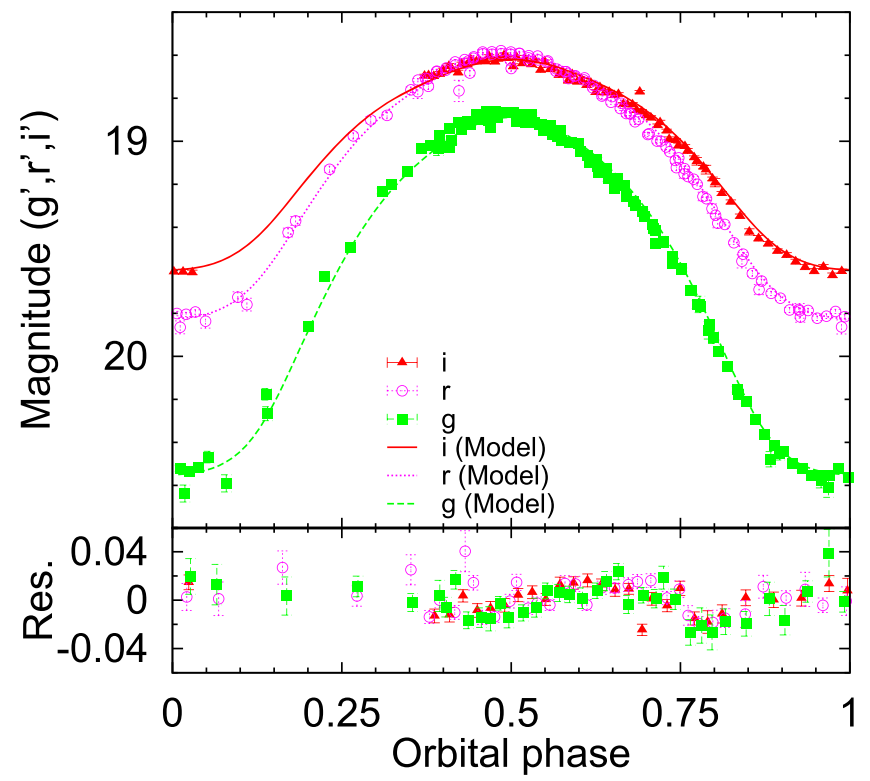

Figure 8. Model fits to the orbital light curves in three bands (top) and residuals (bottom).

averaged into 37, 37, and 28 orbital phase bins, respectively. The Mg I-triplet and Balmer-line RVCs contain 20 data points each (see Section 2.3 for details). Given that there are five different data sets with different numbers of data points, to optimize the fitting procedure, we assigned relative weights to them. After our initial search of the parameter space, which resulted in a good solution, we scaled the uncertainties in each data set (i.e., the LCs and the RVCs) so that the total reduced $\chi^{2}$ of the fit was $\sim 1$ for each data set separately. The MCMC fitting procedure was repeated to produce the final set of parameters, which were used to determine $M_{1}$ and $M_{2}$.

\subsection{Model Results and Comparison with Previous Work}

Our physical model fits simultaneously the Balmer and $\mathrm{Mg}$ I RVCs of J2215 (Figure 7, right) as well as the optical LCs in three bands (Figure 8), with a global reduced $\chi^{2}$ of 1.30 for 126 d.o.f. Small residuals are apparent in the LC fits (especially near $\Phi_{\text {orb }}=0.8$ ) and in the RVC fits (at $\Phi_{\text {orb }}=0.5$ ). We show in Figure 9 the parameter distributions from our XRBCURVE fits to J2215. All model parameters are well constrained, and the overall agreement between the data and model is good. Our best-fit $D=2.9 \pm 0.1 \mathrm{kpc}$ is fully consistent with the $3 \mathrm{kpc}$ value found independently from the MSP dispersion measure (Hessels et al. 2011).

Table 2 presents our best-fit values and their $1 \sigma$ uncertainties, compared to previous studies of J2215. First, as already pointed out by Romani et al. (2015), previous attempts at determining orbital parameters exclusively based on photometric measurements and modeling have been unsuccessful (Schroeder \& Halpern 2014; see also Breton et al. 2013). This is clear, e.g., from the $K_{2}$ velocities predicted by those photometric fit results, inconsistent with our measured values (Table 2). Thus we conclude that, at least in the presence of strong irradiation, dynamical information is required in order to find a reliable orbital solution.

Second, the orbital inclination depends strongly on the temperatures of both sides of the companion. Indeed, a larger temperature contrast between both sides requires a smaller inclination angle to produce the observed peak to peak magnitude difference. Our temperature constraints on the model are taken from quantitative temperature measurements at $\Phi_{\text {orb }}=0.0$ and 0.5 (Section 3.1), and lead to a robustly determined $i=63.9_{-2.7}^{+2.4}$. Therefore, independent constraints on the temperature at different orbital phases are also needed to find a robust solution.

Third, we have shown that a point-source irradiation binary model can fit satisfactorily the J2215 data, in clear contrast with previous results (Romani et al. 2015; Romani \& Sanchez 2016). This discrepancy might also be due to the different temperature constraints, but a more detailed comparison is warranted. In any case, our results show that irradiation from an extended shock is not required to explain the optical properties of J2215. On the other hand, all four models do agree on the filling factor, showing a nearly Roche-lobe-filling companion in J2215. We find an additional nonstellar flux contribution in the range $0.035-0.07 \mathrm{mJy}$ in all three bands $(g, r$, and $i)$, between 2 and 10 times fainter than the companion star (at $\Phi_{\text {orb }}=0$ and 0.5 , respectively). This extra nonvariable flux component, with a rather flat spectral slope, might be due to synchrotron emission from an intrabinary shock, but at present this interpretation remains tentative. We can also rule out the presence of a quiescent disk (as suggested by Schroeder \& Halpern 2014), based on the absence of broad hydrogen and helium emission lines regularly associated with disks.

Our best-fit model predicts a projected rotational velocity for the companion of $V \sin i=103 \pm 1 \mathrm{~km} \mathrm{~s}^{-1}$. Applying an optimal subtraction (Section 2.3) of the $\mathrm{Mg}$ I triplet line region around $\Phi_{\text {orb }}=0$, with a G5 template broadened in steps of $10 \mathrm{~km} \mathrm{~s}^{-1}$ up to $210 \mathrm{~km} \mathrm{~s}^{-1}$, we measure $V \sin i=180 \pm 20 \mathrm{~km} \mathrm{~s}^{-1}$ (with a limb-darkening coefficient $u=0.8$ ) and $V \sin i=165 \pm 15 \mathrm{~km} \mathrm{~s}^{-1}$ (with no limb darkening, $u=0$ ). Thus, taking into account the uncertainty on the amount of limb darkening, our current observational constraints put $V \sin i$ in the range $150-200 \mathrm{~km} \mathrm{~s}^{-1}$. However, better spectral resolution spectra are required to measure this accurately and compare it to our model prediction (our GTC data have a resolution of $160 \mathrm{~km} \mathrm{~s}^{-1}$; Section 2.2). Finally, our best-fit value of $\eta$ implies a very high $L_{\text {irr }}=\left[1.5_{-0.1}^{+0.3}\right.$ ]$\times 10^{35} \mathrm{erg} \mathrm{s}^{-1}$. An irradiating luminosity three times higher than the spin-down energy budget might be explained by, e.g., beaming/anisotropy of the pulsar wind (e.g., Philippov et al. 2015).

The flux distribution along the surface of the companion star is shown in Figure 10, a by-product of our best-fit model of J2215. These maps illustrate the drastic irradiation or "heating" effects of the pulsar wind and high-energy emission on the temperature distribution of the secondary star. Besides producing a strong temperature gradient between opposite sides (which we measure in Section 3.1), such strong heating shifts the effective center of the secondary star ("center of light") away from its center of mass. Because the strength of the lines varies throughout the surface of the star, this shift is different for different absorption lines. This in turn results in significant distortion of the integrated line profiles as well as the corresponding RVCs (e.g., Phillips et al. 1999; Shahbaz et al. 2000). Thus our model provides a natural explanation for the systematic difference in $K$ velocities that we find and report in Section 3.2. 


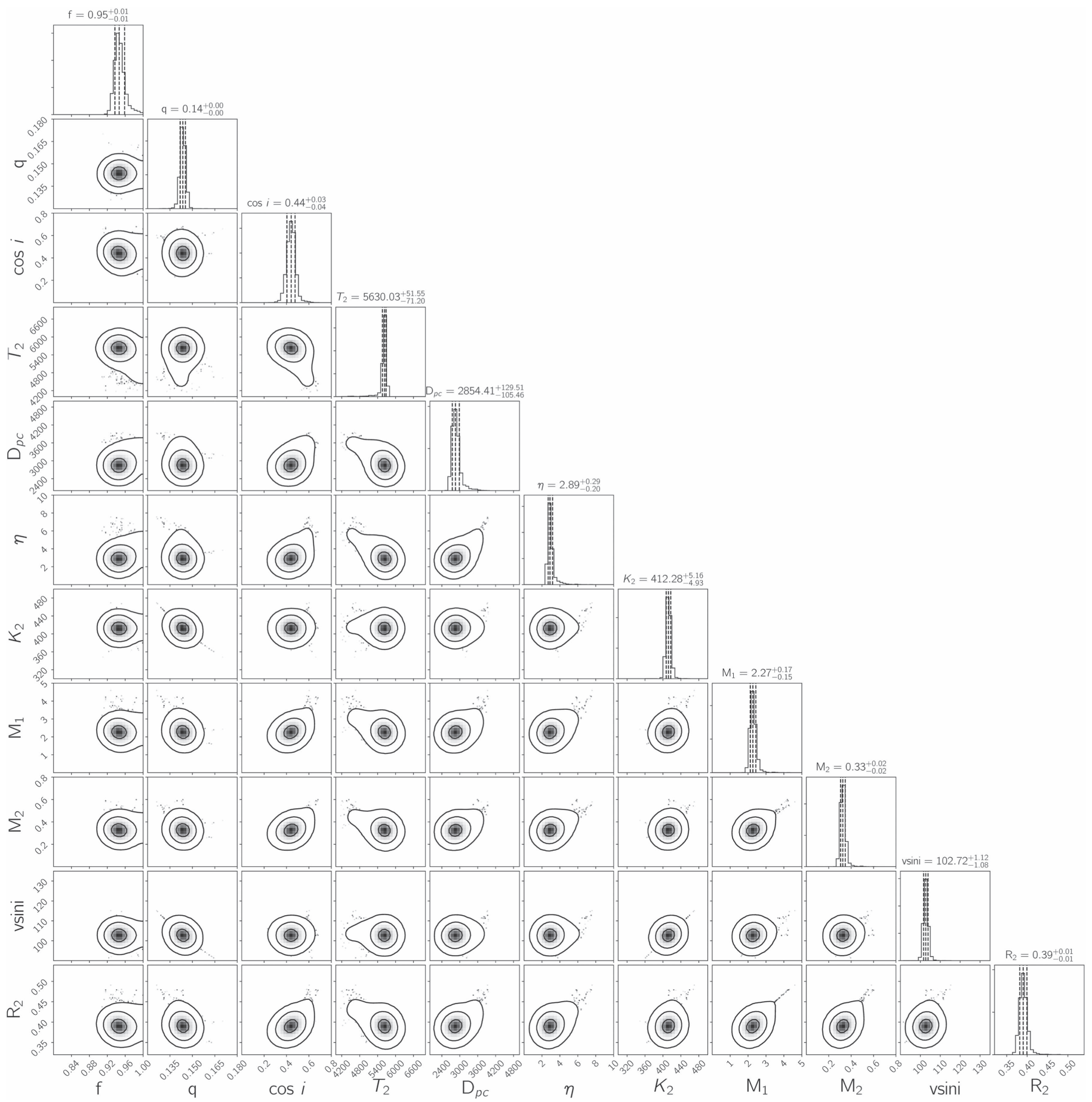

Figure 9. Two-dimensional parameter distributions from our XRBCURVE fits to the RVCs and LCs of J2215. Contours show the $1 \sigma, 2 \sigma$, and $3 \sigma$ confidence regions, and the right panels the projected one-dimensional parameter distributions, together with the $1 \sigma$ errors.

\subsection{On Orbital Phase Shifts and Systematics}

Our model allows for phase shifts in the LC and both RVCs, which may arise physically from, e.g., asymmetric heating of the companion star. The best-fit values for the phase shifts in the Balmer RVC, the Mg I RVC, and the LCs are, respectively, $\Delta \Phi_{\text {Balmer }}=0.018 \pm 0.003, \Delta \Phi_{\mathrm{Mg} \text { I }}=0.0002 \pm 0.0039$ (i.e., consistent with 0 within $1 \sigma$ ), and $\Delta \Phi_{\mathrm{g}}=0.0082 \pm 0.0007$ (where the quoted errors are again $1 \sigma$ statistical). Similar LC phase shifts $\sim 0.01$ have been reported for J2215 (Schroeder \& Halpern 2014; Romani et al. 2015; Romani \& Sanchez 2016), and interpreted as evidence for asymmetric heating from an intrabinary shock.

However, since the model does not take into account errors on the orbital phases, one must consider carefully the systematic uncertainty on $\Phi_{\text {orb }}$ before interpreting such phase shifts. If we used the pulsar timing reference epoch to calculate $\Phi_{\text {orb }}$ (which dates from 2009 December 21, from Abdo et al. 2013), we should include the orbital period derivative $\dot{P}_{\text {orb }}$ (not doing so would yield a propagated uncertainty to our 2014 August 02 epoch of 0.02 orbital cycles). But assuming a constant $\dot{P}_{\text {orb }}$ over a 5 year timespan is problematic, since 

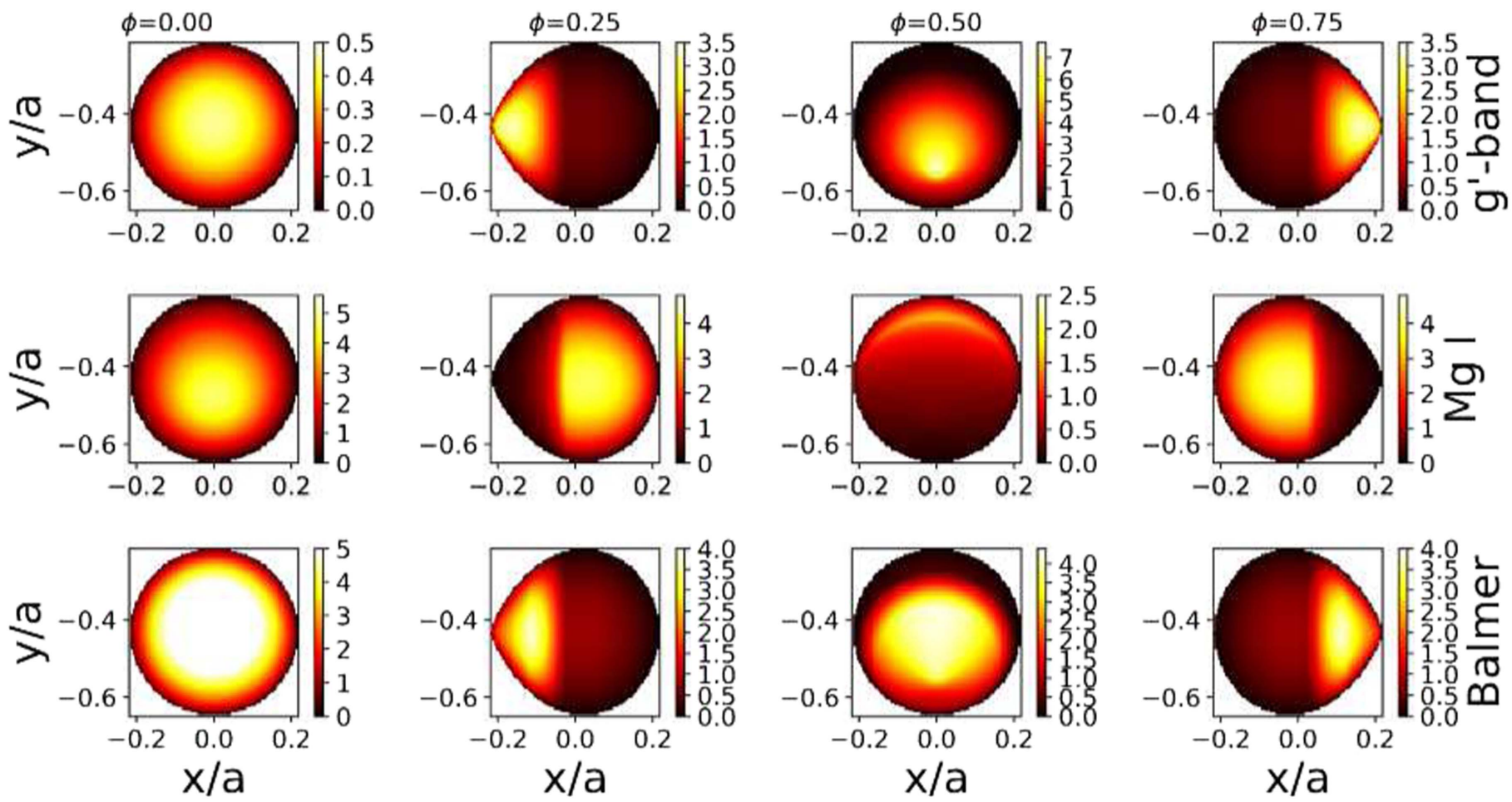

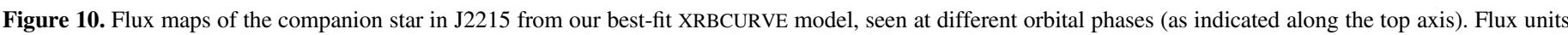

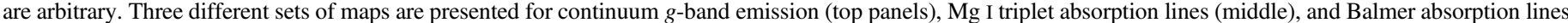

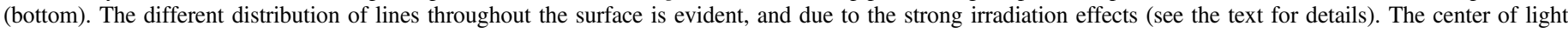
depends strongly on the line or spectral range chosen.

redbacks and black widows are known to show large, erratic changes in $\dot{P}_{\text {orb }}$ on timescales of years (e.g., Archibald et al. 2013). Instead, we use our own spectroscopic reference time (T0, see Section 3.2) with a systematic uncertainty of 0.0008 days, which corresponds to a systematic uncertainty on $\Phi_{\text {orb }}$ of $\delta \Phi_{\text {orb }}=0.0046$ cycles.

In light of this, the only phase shift that we deem (marginally) significant is $\Delta \Phi_{\text {Balmer, which is larger than } 0 \text { at }}$ the $3.3 \sigma$ confidence level (this shift can be seen by close inspection of Figure 7). Thus we conclude that, even if orbital phase shifts are necessary to allow a good statistical fit to the data, their significance and interpretation depend critically on the systematics of the orbital ephemeris. From our data and model of J2215, after quantifying the systematic error on our orbital phases, we find one relatively small phase shift in the Balmer-line RVC and thus only marginal evidence for heating asymmetries.

\section{Discussion}

\subsection{Measuring Masses: An Empirical K Correction}

In a careful study of the strongly irradiated companion star of PSR J2215+5135, we find that the radial velocities depend systematically on the spectral features and spectral range used to measure them (Section 3.2). Thanks to the high-quality GTC optical spectra, we show that magnesium lines yield semiamplitudes of the RVC that are always higher than those inferred from hydrogen Balmer lines or from an undetermined blend of lines. We argue that this new systematic effect arises from the extreme contrast between the the cold and heated sides of the companion star, for which we measure temperatures of $T_{\mathrm{N}}=5660_{-380}^{+260} \mathrm{~K}$ and $T_{\mathrm{D}}=8080_{-280}^{+470} \mathrm{~K}$, respectively (Section 3.1). Under these circumstances, Balmer lines trace the hot face of the companion star of $\mathbf{J} 2215$ in its orbit around the pulsar, while $\mathrm{Mg}$ I triplet lines trace its cold, unperturbed face.

We modeled both RVCs and the optical LCs in three bands, and found the center of mass velocity to be $K_{2}=412.3 \pm$ $5.0 \mathrm{~km} \mathrm{~s}^{-1}$ (Section 4). Because the center of light of the $\mathrm{Mg} \mathrm{I}$ lines yields higher velocities $\left(K_{\mathrm{Mg}}=K_{2}+\Delta K, \Delta K>0\right)$, it is slightly shifted outwards from the center of mass of the companion. Using the simple expression for the center-of-light displacement $\Delta R / a=\Delta K /\left(K_{2}(1+q)\right)$ (Wade \& Horne 1988) and our best-fit orbital parameters (Table 2), we estimate $\Delta R_{\mathrm{Mg} I} / R_{\mathrm{RL} 2} \simeq+0.11$ (i.e., about $11 \%$ of the Roche-lobe radius of the companion). Balmer lines, on the other hand, suffer from a stronger inwards displacement of the center of light, which can yield to systematic errors in dynamical mass measurements if not corrected. We estimate this displacement as above using the measured $K_{\mathrm{Balmer}}$, and find $\Delta R_{\mathrm{Balmer}} / R_{\mathrm{RL} 2} \simeq-0.24$ (i.e., a $24 \%$ shift relative to $R_{\mathrm{RL} 2}$ ).

Given the large $(\simeq 10 \%)$ differences in the measured $K$ values and the $M_{1} \propto K^{3}$ relation, this has important consequences for the measured pulsar masses. For instance, using the different measured $K$ values and our best-fit orbital solution (Section 4) yields inconsistent values for the neutron star mass: $M_{1}=1.88 \pm 0.16 M_{\odot}\left(\right.$ from $\left.K_{\text {Balmer }}=382.8 \pm 4.7 \mathrm{~km} \mathrm{~s}^{-1}\right)$ and $M_{1}=2.49 \pm 0.23 M_{\odot}\left(\right.$ from $\left.K_{\mathrm{Mg}}=420.2 \pm 6.2 \mathrm{~km} \mathrm{~s}^{-1}\right)$. Thus we find that, in the presence of strong irradiation, the systematic error on $K$ may be equally or more important than the uncertainty on the orbital inclination, $i$.

To circumvent this and drastically reduce the systematics on $K$ measurements, we have put forward a new method: we measure $K$ velocities using different sets of lines in order to "bracket" the center-of-mass velocity of the companion star. 
We have deemed as optimal the Balmer and $\mathrm{Mg}$ I triplet lines (Section 2.3) that provide a lower and an upper limit on $K_{2}$, respectively. These could, in principle, be replaced by other sets of lines that trace the movement of both the cold/dark and irradiated/bright sides of the star. While the traditional " $\mathrm{K}$ correction" relies on model assumptions or on the transient nature of irradiation in some systems (Section 5.2), our method provides a direct way of quantifying this correction from the same spectra of the irradiated companion star.

To our knowledge, the only similar studies in the literature involve a combination of emission and absorption lines in white dwarf (WD) binaries (e.g., Parsons et al. 2010; Rodríguez-Gil et al. 2015). Our method relies exclusively on absorption lines from the star's atmosphere, thus eluding the uncertainty sometimes associated with the exact site where Balmer/He/ Bowen emission lines are formed. This "empirical $K$ correction" is of particular relevance for the emerging population of MSPs in compact binaries, and it should also be applicable in the broader context of semidetached binaries with strong irradiation/heating effects.

In summary, our findings show that metallic lines, in general, and $\mathrm{Mg}$ I lines, in particular, offer a much less distorted view of the center of mass of the secondary star, opening a new way to measure masses in strongly irradiated compact binary MSPs. In the relevant temperature and spectral type range for J2215 and most redback and black widow companions (spectral types A through M), Balmer lines are more sensitive to temperature than $\mathrm{Mg}$ I triplet lines (see the Appendix, Figure 13). Indeed, when going from a spectral type A5 to a G5, the EW of Balmer lines decreases by a factor of $\simeq 7(48 \rightarrow 7 \AA)$, whereas the EW of $\mathrm{Mg} \mathrm{I}$ triplet lines increases by a smaller factor $\simeq 3$ $(1.52 \rightarrow 4.75 \AA)$. This may explain why the effects of irradiation are more drastic on Balmer absorption lines than on metallic lines.

\subsection{A Broader Look at Irradiation: $K$ Correction and Deep Heating}

Irradiation in compact binaries has been studied in the context of black hole and neutron star LMXBs as well as WD binaries (dwarf novae, DN, post-common envelope binaries, PCEBs, and asynchronous polars, AP). In those systems, irradiation proceeds mostly through X-ray and UV photons from a hot WD or innermost accretion disk. The ratio of the maximum irradiating flux at the companion's surface (near the inner Lagrangian point) over the companion's intrinsic unperturbed flux provides a good way to quantify the importance of irradiation in close binaries: $f_{\text {irr }}=\frac{L_{\text {irr }} R_{2}^{2}}{L_{2} a^{2}}$. The effects of irradiation on the measured $K$ velocities, on the other hand, are typically parameterized in terms of the so-called $K$ correction, which we define as the ratio between observed and center-of-mass $K$ values: $f_{K}=\frac{K_{\mathrm{obs}}}{K_{2}}$. This correction is estimated in the literature in a number of different ways, e.g., by comparing outburst and quiescence $K$ values (Hessman et al. 1984) or by simulating and fitting RVCs with irradiation models (Wade \& Horne 1988; Phillips et al. 1999; MuñozDarias et al. 2005).

In some cases (compact LMXBs in outburst, very hot WDs), chromospheric/fluorescent emission lines are formed on the inner face of the companion star, leading to a lower limit on the center of mass velocity semi-amplitude $K_{2}$ (i.e., $f_{K}<1$ ). This is the case of Bowen fluorescence lines in LMXBs. It is interesting to compare $\mathbf{J} 2215$ with the accreting millisecond pulsars SAX J1808.4-3658 and XTE J1814-338, with Bowenline outburst $K$ corrections of $f_{K}=0.90$ and 0.81 , respectively (Cornelisse et al. 2009; Wang et al. 2017). These are analogous to (and possibly evolutionary precursors of black widow and redback MSPs. Assuming an irradiating luminosity in outburst $L_{X} \sim 10^{36} \mathrm{erg} \mathrm{s}^{-1}$, we estimate their maximum ratio of irradiating to intrinsic flux and find extreme values, $f_{\text {irr }} \sim$ $10^{3}-10^{4}$. For the persistent atoll sources $4 \mathrm{U} 1636-536$ and 4U 1735-444, with $f_{K} \simeq 0.77$ (Casares et al. 2006), we estimate even higher $f_{\text {irr }} \sim 10^{5}$.

Balmer/He I emission lines in WD binaries are also associated with strong, yet less extreme, irradiation. The AP V1500Cyg, for instance, has an estimated $f_{K}=0.65$ (Balmer and He II lines, Horne \& Schneider 1989) and we find $f_{\text {irr }} \sim 50$ (using the stellar parameters in Schmidt et al. 1995). Similarly, we find $f_{\text {irr }} \sim 140$ for the PCEB NNSer, with a reported $f_{K}=0.88$ (Balmer lines, Parsons et al. 2010). This suggests that the irradiating flux must be at least 10 times higher than the intrinsic stellar flux in order to form emission lines on the heated face.

In other cases (DNe and BHCs in outburst), absorption lines are partially quenched on the inner face of the companion due to irradiation, so that the effective center of light for these lines is shifted toward the outer face and they provide an upper limit on $K_{2}$ (i.e., $f_{K}>1$ ). Even though stellar atmospheres with external heating are poorly understood, the reduced absorption line strength is often attributed to the reduced vertical temperature gradient in the presence of an external UV/Xray photon flux. This is the case of the WD binaries ZCha, UGem, and SSCyg, with $f_{K}$ estimated at 1.03, 1.04, and 1.26, respectively (Hessman et al. 1984; Wade \& Horne 1988; Friend et al. 1990). For these systems, we find mild irradiation, with estimated $f_{\text {irr }}$ values in the range $0.1-3$. The BHC GRO J165540 , on the other hand, with $f_{K} \simeq 1.16$, has a relatively luminous F6IV companion star that also leads to a mild $f_{\text {irr }} \sim 7$ (Orosz \& Bailyn 1997; Phillips et al. 1999).

In our case (J2215) and in compact binary MSPs, in general, the relativistic pulsar wind and gamma-ray emission are the dominant sources of irradiation. These feature typical spin-down luminosities $\dot{E}=10^{34}-10^{35} \mathrm{erg} \mathrm{s}^{-1}$ and gamma-ray luminosities $\left(L_{\gamma}\right)$ about 10 times lower. Their X-ray luminosities are two to five orders of magnitude lower than $\dot{E}$ and are thus less important in terms of irradiation $\left(L_{\mathrm{X}}=10^{30}-10^{32} \mathrm{erg} \mathrm{s}^{-1}\right.$; Gentile et al. 2014; Linares 2014). Indeed, J2215 has $L_{\mathrm{X}}=1.2 \times 10^{32} \mathrm{erg} \mathrm{s}^{-1}$ $(0.5-10 \mathrm{keV}), L_{\gamma}=1.4 \times 10^{34} \mathrm{erg} \mathrm{s}^{-1}(0.1-100 \mathrm{GeV})$, and $\dot{E}=$ $5.3 \times 10^{34} \mathrm{erg} \mathrm{s}^{-1}$ (Linares 2014; Acero et al. 2015; Breton et al. 2013, respectively; for a $2.9 \mathrm{kpc}$ distance). Our measured radial velocity amplitudes $K_{\mathrm{Balmer}}$ and $K_{\mathrm{Mg}}$, together with the center-of-mass velocity from our best-fit model $\left(K_{2}\right)$ imply $K$ correction factors for $\mathbf{J} 2215$ of $f_{K}=0.928$ and $f_{K}=1.019$, respectively. In other words, the $K$ values measured using Balmer and $\mathrm{Mg}$ I lines are $7.2 \%$ lower and $1.9 \%$ higher, respectively, than the true center-of-mass $K_{2}$.

For comparison, the $K$ correction inferred by van Kerkwijk et al. (2011) for the black widow pulsar PSR B1957+20 was close to 8\%: $f_{K}=0.918$ (where their $K_{\text {obs }}$ was measured using a slightly wider spectral range and a G2 template). Thus, $K$ corrections may be similarly important in black widow and redback binaries. This can be understood qualitatively from the two terms entering $f_{\text {irr }}$, which have opposite trends: $R_{2}^{2} / a^{2}$ is lower in black widows (lower irradiating flux due to smaller 


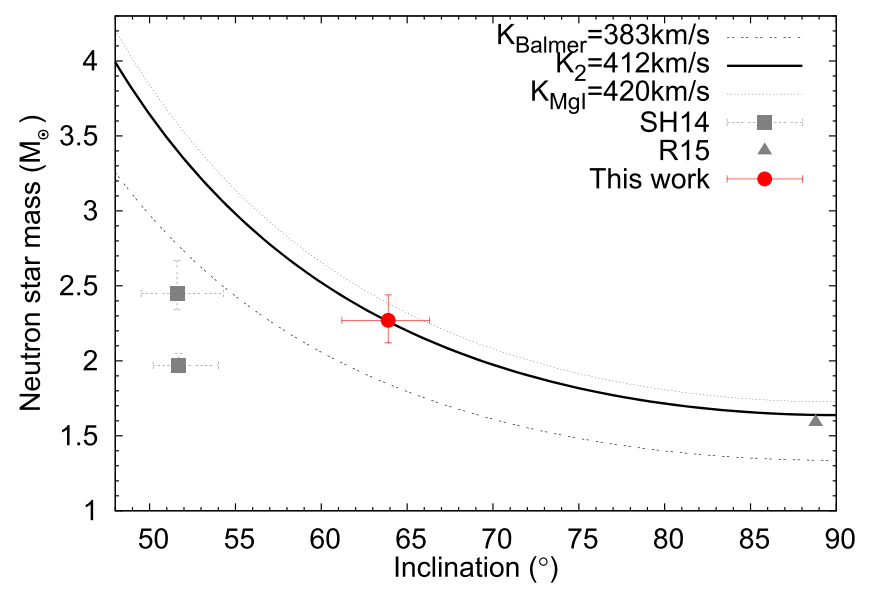

Figure 11. Neutron star mass measurements in J2215, shown vs. orbital inclination. Dotted and dashed lines show the effects of using different sets of absorption lines to measure velocities (Sections 3.2 and 5.1). The thick solid line shows our model $K_{2}$ (Section 4). Our best-fit orbital solution (filled red circle) is compared with previous work (Schroeder \& Halpern 2014; Romani et al. 2015; filled squares and triangles, respectively).

solid angle), but $L_{\text {irr }} / L_{2}$ is higher compared to redbacks (as the black widow companions are less luminous).

Using our orbital solution (which gives $R_{\mathrm{RL} 2} / a=0.23$ ), our best-fit value of $L_{\text {irr }} \simeq 3 \times \dot{E}$, and a companion luminosity $L_{2} \simeq$ $5.3 \times 10^{32} \mathrm{erg} \mathrm{s}^{-1}$ (from our best-fit $T_{2}=5630 \mathrm{~K}$ and $R_{2}=$ $0.39 R_{\odot}$ ), we estimate $f_{\text {irr }} \sim 15$. In other words, the irradiating flux at the companion's heated face is up to 15 times higher than the intrinsic stellar flux. Compared with UV and X-ray photons, Gamma-ray photons and relativistic particles from the pulsar wind are expected to penetrate deeper into the companion atmosphere. This leads to deep/internal heating of the companion's inner face, so it is not surprising to find no emission lines in $\mathrm{J} 2215$, and no quenching of absorption lines either (Figure 4).

\subsection{The Mass of PSR J2215+5135}

We have argued that our "empirical $K$ correction" removes a critical systematic uncertainty in irradiated systems: the difference between center of light and center of mass of the companion. In $\mathrm{J} 2215$, our $K_{2}=412.3 \pm 5.0 \mathrm{~km} \mathrm{~s}^{-1}$ yields a mass function of $1.2 M_{\odot}$, an absolute lower limit on the pulsar mass. Combined with the tight constraints on $q$ (Section 4; Abdo et al. 2013), this implies a minimum neutron star mass of $1.6 M_{\odot}$. Thus we find that $\mathrm{J} 2215$ contains a neutron star more massive than the "canonical" $1.4 M_{\odot}$ double neutron stars, adding to the growing number of such systems (Demorest et al. 2010; Antoniadis et al. 2013; Özel \& Freire 2016).

Furthermore, we have shown that J2215 could harbor the most massive pulsar known to date. Our best-fit model yields a very massive neutron star with $M_{1}=2.27_{-0.15}^{+0.17} \quad M_{\odot}$, and a wellconstrained inclination of $i=63.9_{-2.7}^{+2.4}$ (see Figure 11). This is more massive than the previous well-established record holder (2.01 $M_{\odot}$, Antoniadis et al. 2013), at the $97 \%$ confidence level. The results of Romani et al. (2015) and Romani \& Sanchez (2016) are clearly at odds, although their nearly edge-on model (with a neutron star mass of $1.6 M_{\odot}$ ) has no uncertainties reported and admittedly fails to describe the data. In a previous study of the original black widow pulsar, van Kerkwijk et al. (2011) found a pulsar mass that was similarly high $\left(2.40 \pm 0.12 M_{\odot}\right)$. The main advances of the method we have presented here are (i) an empirical $K$ correction, based on radial velocities measured with two different sets of lines (Section 3.2) and (ii) independent constraints on the temperature imposed on the model, based on absorption line strengths (Section 3.1). We argue that these two new advances make our results more robust compared to previous work (van Kerkwijk et al. 2011; Romani et al. 2015; Romani \& Sanchez 2016). There may still be unknown or highly uncertain systematic effects; however, biasing the best-fit model inclination and thus the dynamical mass measurements (see, e.g., the discussion in van Kerkwijk et al. 2011, their Section 4).

We therefore conclude that, if confirmed with an independent measurement of the orbital inclination, the massive neutron star in J2215 may place new constraints on the equation of state at supranuclear densities. This would push the limits of the most massive neutron stars in our Galaxy, setting a lower limit of $2.3 M_{\odot}$ on their maximum mass. Since particle interactions in the core provide the pressure necessary to halt its collapse, the maximum mass of a neutron star places independent constraints on how these particles interact (Lattimer \& Prakash 2007). For instance, exotic forms of matter such as hyperons or deconfined quarks have been proposed to exist in the central parts of a neutron star, yet they can hardly account for a neutron star as massive as the one we find in J2215 (see also Özel \& Freire 2016).

With new Galactic MSPs being currently discovered at a rate of 10-30 per year (Lorimer 2018), the neutron star mass range will be explored further in the next decade, and is likely to continue widening. We have shown here that in the study of strongly irradiated pulsar companions, a controlled measurement of temperatures and velocities throughout the orbit is possible with current instruments and key to finding a robust dynamical solution. Our novel technique, which combines velocity measurements with different absorption lines, temperature measurements, and physical modeling of the binary, should provide a path forward for dynamical mass measurements in this growing population.

\section{Summary and Conclusions}

1. We have identified for the first time, and thanks to GTC's large collecting area, absorption lines from both sides of the irradiated companion star to PSR J2215+5135. We show that Mg I triplet lines effectively trace the unheated "dark side" of the companion, while hydrogen Balmer lines trace its irradiated side. We are therefore able to bracket the center of mass velocity, placing both an upper and a lower limit on $K_{2}$. This removes the systematic uncertainty on $K_{2}$ in strongly irradiated systems due to the displacement of the center of light, traditionally incorporated in the so-called " $K$ correction."

2. We argue that, beyond light-curve modeling, accurate mass measurements in strongly irradiated binary systems require (i) radial velocities, preferably measured using metallic lines, and (ii) robust constraints on the temperatures of both sides of the companion.

3. In particular, we find that the semi-amplitude of the RVC of J2215 measured with Mg I lines is systematically higher than that measured with Balmer lines, by $10 \%$.

4. We measure temperatures for the cold and hot sides of $T_{\mathrm{N}}=5660_{-380}^{+260} \mathrm{~K}$ and $T_{\mathrm{D}}=8080_{-280}^{+470} \mathrm{~K}$, respectively. 
5. By modeling jointly both RVCs and the light curves in three bands, while imposing the temperature constraints above, we find that $\mathrm{J} 2215$ has (i) a center-of-mass $K$ velocity of $K_{2}=412.3 \pm 5.0 \mathrm{~km} \mathrm{~s}^{-1}$; (ii) an inclination $i=63^{\circ} .9_{-2.7}^{+2.4}$; (iii) an apparent irradiating luminosity three times higher than its spin-down luminosity; and (iv) a companion close to filling its Roche lobe (filling factor $\left.0.95_{-0.01}^{+0.01}\right)$.

6. Our physical modeling can reproduce the measured fluxes and velocities without invoking extended irradiation, and yields only marginal evidence for asymmetric heating (in the form of orbital phase shifts).

7. We thereby find that J2215 hosts a main-sequence G5 companion with $M_{2}=0.33_{-0.02}^{+0.03} M_{\odot}$ and a very massive neutron star with $M_{1}=2.27_{-0.15}^{+0.17} M_{\odot}$.

8. Pending independent confirmation of the orbital inclination, our results strongly suggest that the maximum neutron star mass is at least $\sim 2.3 M_{\odot}$.

Based on observations made with the GTC, WHT, and IAC80 telescopes operated by IAC and ING in the Spanish Observatories of el Roque de los Muchachos (La Palma) and el Teide (Tenerife) under regular, service, and DDT programs. We thank C. Fariña, P. Chinchilla, R. Karjalainen, A. CabreraLavers, A. Oscod, and R. Corradi for support during these observations, and the IAC director R. Rebolo for granting the DDT. This work was supported by the Spanish Ministry of Economy and Competitiveness (MINECO) under the grant AYA2013-42627. IRAF is distributed by the NOAO, operated by AURA under cooperative agreement with NSF. We thank T. Marsh for the use of MOLLY and ULTRACAM, and acknowledge the use of data from the UVES Paranal Observatory Project (ESO DDT Program ID 266.D-5655). We appreciate discussions with R. Alonso, R. Breton, P. Charles, J. José T. Muñoz-Darias, and P. Rodríguez-Gil during different stages of this work. M.L. is supported by EU's Horizon 2020 programme through a Marie Sklodowska-Curie Fellowship (grant No. 702638).

\section{Appendix \\ A Stellar Spectral Library for Temperature and Radial Velocity Measurements}

We built a library of main-sequence stellar spectra (or "templates") in order to measure spectral types (temperatures) and radial velocities (Section 2.3). We used 33 VLT-UVES spectra from the Paranal Observatory Project (Bagnulo et al. 2003, UVES-POP), initially rebinned to a $0.2 \AA$ resolution, which cover the $\sim 3000-10000 \AA$ range. We normalized them to their continuum level by fitting a spline function, and excluded the gaps between echelle orders.

We then subtracted each template's radial velocity, measured by cross-correlating the $\mathrm{H} \alpha$ and $\mathrm{H} \beta$ line profiles with a Gaussian with FWHM $=100 \mathrm{~km} \mathrm{~s}^{-1}$, or two such Gaussians separated by $200 \mathrm{~km} \mathrm{~s}^{-1}$ in the broad line cases. These radial velocities were in good agreement with the values listed in the SIMBAD database. The resulting spectra are shown in Figure 12, broadened to match the spectral resolution of the GTC-OSIRIS spectra presented herein $\left(160 \mathrm{~km} \mathrm{~s}^{-1}\right.$; Section 2.2). We also calculated the EW of absorption lines in the templates, shown in Figure 13, which we use in our modeling of the Balmer-line and Mg I-triplet radial velocities (Section 4).

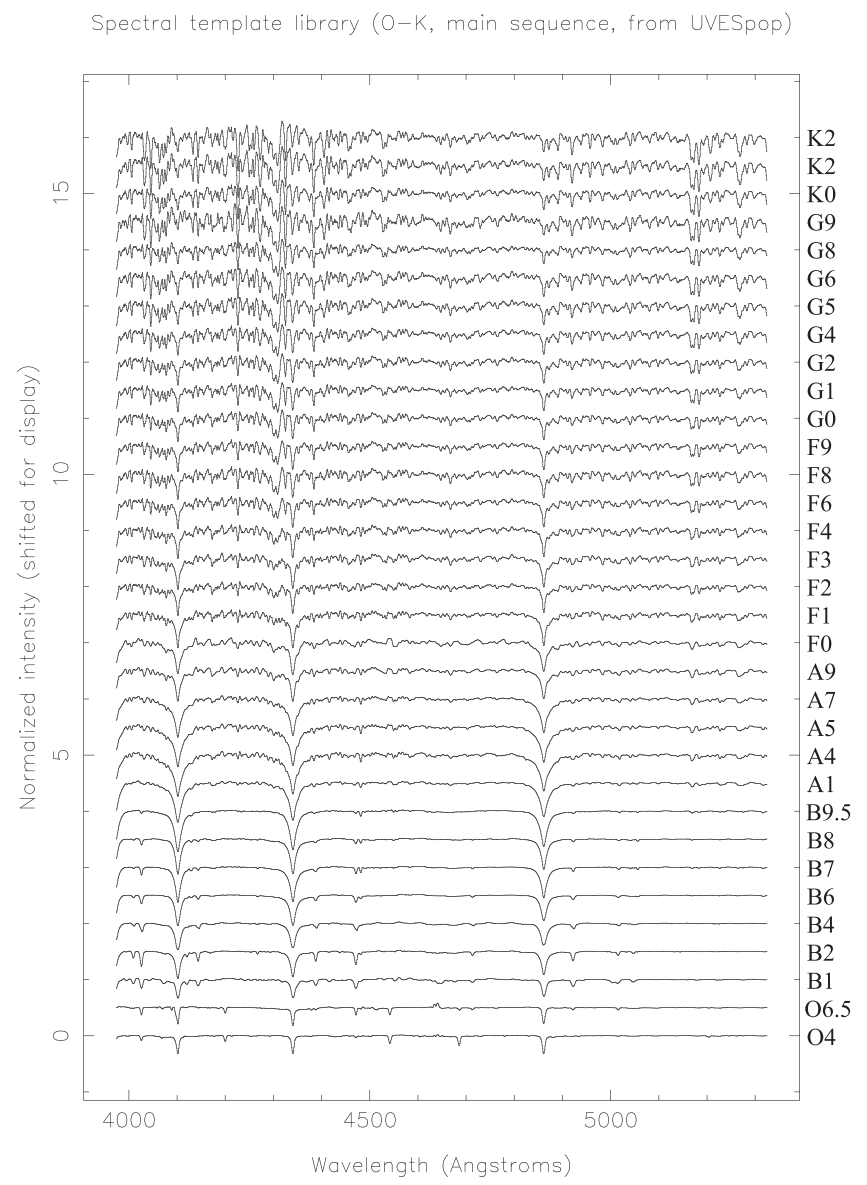

Figure 12. Template library adapted from UVES-POP (Bagnulo et al. 2003) for temperature and radial velocity measurements. Normalized intensities are shifted for display, and the spectral type is noted along the right axis.

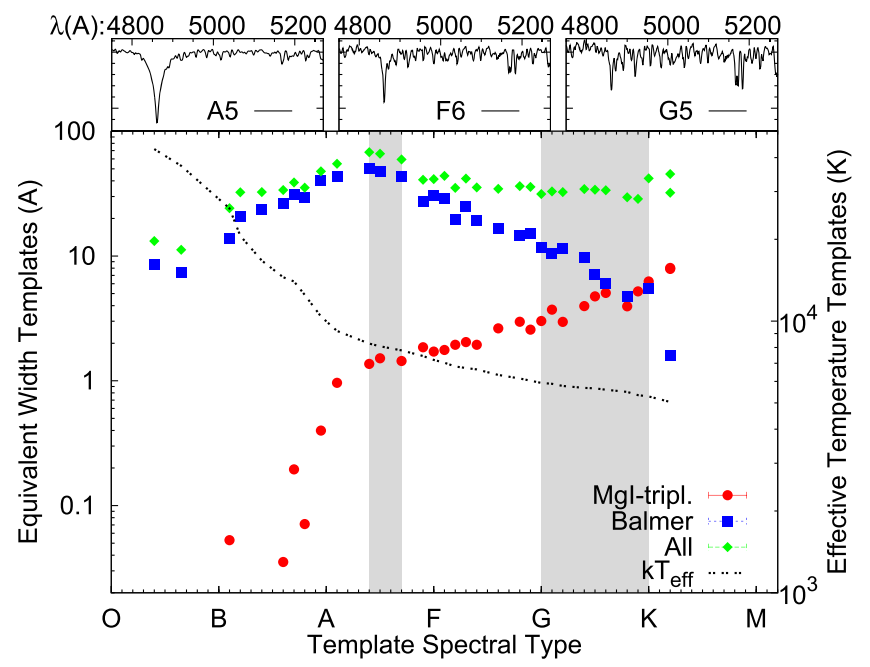

Figure 13. EW of absorption lines from our template spectra, in the three ranges used for radial velocity measurements: (i) Balmer lines (blue squares); (ii) Mg I triplet (red circles); and the full GTC-OSIRIS range (green triangles). The dotted line shows the template temperature (right vertical axis; from Pecaut \& Mamajek 2013). Three representative spectra are shown in the top panels, within the $\mathrm{H} \beta$ and $\mathrm{Mg}$ I-triplet line region.

\section{ORCID iDs}

M. Linares (10) https://orcid.org/0000-0002-0237-1636

J. Casares (iD https://orcid.org/0000-0001-5031-0128 


\section{References}

Abdo, A. A., Ajello, M., Allafort, A., et al. 2013, ApJS, 208, 17 Acero, Ackermann, M., Ajello, M., et al. 2015, F, ApJS, 218, 23 Antoniadis, J., Freire, P. C. C., Wex, N., et al. 2013, Sci, 340, 448 Archibald, A. M., Kaspi, V. M., Hessels, J. W. T., et al. 2013, ApJ, submitted (arXiv:1311.5161)

Archibald, A. M., Stairs, I. H., Ransom, S. M., et al. 2009, Sci, 324, 1411

Atwood, W. B., Abdo, A. A., Ackermann, M., et al. 2009, ApJ, 697, 1071

Bagnulo, S., Jehin, E., Ledoux, C., et al. 2003, Msngr, 114, 10

Breton, R. P., van Kerkwijk, M. H., Roberts, M. S. E., et al. 2013, ApJ, 769, 108

Casares, J., Cornelisse, R., Steeghs, D., et al. 2006, MNRAS, 373, 1235

Claret, A. 2000, A\&A, 363, 1081

Cornelisse, R., D’Avanzo, R., Muñoz-Darias, T., et al. 2009, A\&A, 495, L1

Crawford, F., Lyne, A. G., Stairs, I. H., et al. 2013, ApJ, 776, 20

de Martino, D., Belloni, T., Falanga, M., et al. 2013, A\&A, 550, A89

Demorest, P. B., Pennucci, T., Ransom, S. M., Roberts, M. S. E., \& Hessels, J. W. T. 2010, Natur, 467, 1081

Friend, M. T., Martin, J. S., Connon-Smith, R., \& Jones, D. H. P. 1990, MNRAS, 246, 637

Gentile, P. A., Roberts, M. S. E., McLaughlin, M. A., et al. 2014, ApJ, 783, 69

Gregory, P. C. 2005, Bayesian Logical Data Analysis for the Physical Sciences: A Comparative Approach with "Mathematica" Support (Cambridge: Cambridge Univ. Press)

Hauschildt, P. H., Allard, F., \& Baron, E. 1999, ApJ, 512, 377

He, C., Ng, C.-Y., \& Kaspi, V. M. 2013, ApJ, 768, 64

Hessels, J. W. T., Roberts, M. S. E., McLaughlin, M. A., et al. 2011, in AIP Conf. Ser. 1357, Radio Pulsars, ed. M. Burgay et al. (Melville, NY: AIP), 40

Hessman, F. V., Robinson, E. L., Nather, R. E., \& Zhang, E.-H. 1984, ApJ, 286,747

Horne, K., \& Schneider, D. P. 1989, ApJ, 343, 888

Jordi, C., Høg, E., Brown, A. G. A., et al. 2006, MNRAS, 367, 290

Kalberla, P. M. W., Burton, W. B., Hartmann, D., et al. 2005, A\&A, 440, 775

Kaplan, D. L., Bhalerao, V. B., van Kerkwijk, M. H., et al. 2013, ApJ, 765, 158
Lattimer, J. M., \& Prakash, M. 2007, PhR, 442, 109

Linares, M. 2014, ApJ, 795, 72

Lorimer, D. 2018, Galactic Millisecond Pulsars, http://astro.phys.wvu.edu/ GalacticMSPs

Lucy, L. B. 1967, ZA, 65, 89

Marsh, T. R. 1989, PASP, 101, 1032

Marsh, T. R., Robinson, E. L., \& Wood, J. H. 1994, MNRAS, 266, 137 Muñoz-Darias, T., Casares, J., \& Martìnez-Pais, I. G. 2005, ApJ, 635, 502

Orosz, J. A., \& Bailyn, C. D. 1997, ApJ, 477, 876

Özel, F., \& Freire, P. 2016, ARA\&A, 54, 401

Papitto, A., Ferrigno, C., Bozzo, E., et al. 2013, Natur, 501, 517

Parsons, S. G., Marsh, T. R., Copperwheat, C. M., et al. 2010, MNRAS, 402, 2591

Pecaut, M. J., \& Mamajek, E. E. 2013, ApJS, 208, 9

Philippov, A. A., Spitkovsky, A., \& Cerutti, B. 2015, ApJL, 801, L19

Phillips, S. N., Shahbaz, T., \& Podsiadlowski, P. 1999, MNRAS, 304, 839

Predehl, P., \& Schmitt, J. H. M. M. 1995, A\&A, 293, 889

Rodríguez-Gil, P., Shahbaz, T., Marsh, T. R., et al. 2015, MNRAS, 452, 146

Romani, R. W., Graham, M. L., Filippenko, A. V., \& Kerr, M. 2015, ApJL, 809, L10

Romani, R. W., \& Sanchez, N. 2016, ApJ, 828, 7

Romani, R. W., \& Shaw, M. S. 2011, ApJL, 743, L26

Schlegel, D. J., Finkbeiner, D. P., \& Davis, M. 1998, ApJ, 500, 525

Schmidt, G. D., Liebert, J., \& Stockman, H. S. 1995, ApJ, 441, 414

Schroeder, J., \& Halpern, J. 2014, ApJ, 793, 78

Shahbaz, T., Groot, P., Phillips, S. N., et al. 2000, MNRAS, 314, 747

Shahbaz, T., Linares, M., \& Breton, R. P. 2017, MNRAS, 472, 4287

Shahbaz, T., Thorstensen, J. R., Charles, P. A., \& Sherman, N. D. 1998, MNRAS, 296, 1004

Shahbaz, T., Zurita, C., Casares, J., et al. 2003, ApJ, 585, 443

van Kerkwijk, M. H., Breton, R. P., \& Kulkarni, S. R. 2011, ApJ, 728, 95

Vrugt, J. A. 2016, Environ. Modelling Softw., 75, 273

Wade, R. A., \& Horne, K. 1988, ApJ, 324, 411

Wang, L., Steeghs, D., Casares, J., et al. 2017, MNRAS, 466, 2261 